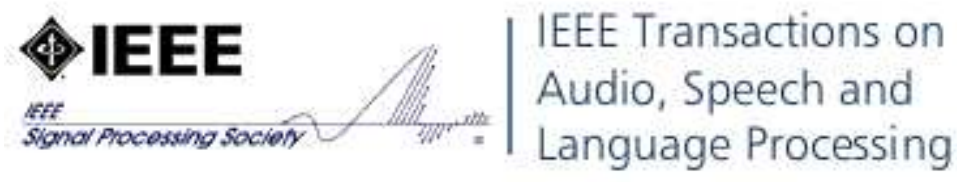

\title{
Numerical Methods for a Non-Linear Impact Model: a Comparative Study with Closed-Form Corrections
}

\begin{tabular}{|r|l|}
\hline Journal: & Transactions on Audio, Speech and Language Processing \\
\hline Manuscript ID: & T-ASL-02612-2009.R1 \\
\hline Manuscript Type: & Regular Paper \\
\hline Date Submitted by the \\
Author: & 25-Jun-2010 \\
\hline Complete List of Authors: & $\begin{array}{l}\text { Papetti, Stefano; University of Verona, Dept. of Computer Science } \\
\text { Avanzini, Federico; University of Padova, Dept. of Information } \\
\text { Engineering } \\
\text { Rocchesso, Davide; IUAV University of Venice, Dep. of Arts and } \\
\text { Industrial Design }\end{array}$ \\
\hline EDICS: & $\begin{array}{l}\text { AUD-AUMM Audio for Multimedia < AUDIO AND } \\
\text { ELECTROACOUSTICS, AUD-ANSY Audio Analysis and Synthesis < } \\
\text { AUDIO AND ELECTROACOUSTICS }\end{array}$ \\
\hline
\end{tabular}

\section{s ScholarONE" \\ Manuscript Central}


Numerical Methods for a Non-Linear Impact Model: a Comparative Study with Closed-Form

\title{
Corrections
}

\author{
Stefano Papetti*, Federico Avanzini, and Davide Rocchesso
}

\begin{abstract}
A physically-based impact model - already known and exploited in the field of sound synthesis is studied using both analytical tools and numerical simulations. It is shown that the Hamiltonian of a physical system composed of a mass impacting on a wall can be expressed analytically as a function of the mass velocity during contact. Moreover, an efficient and accurate approximation for the mass outbound velocity is presented, which allows to estimate the Hamiltonian at the end of the contact. Analytical results are then compared to numerical simulations obtained by discretizing the system with several numerical methods. It is shown that, for some regions of the parameter space, the trajectories of the discretized systems may significantly drift from the analytically-derived curves. Two approaches, based on enforcing numerical energy consistency, are then proposed to improve the accuracy of numerical simulations.
\end{abstract}

\section{Index Terms}

Real time systems, simulation.

\section{INTRODUCTION}

Physical models of impacts between objects are ubiquitous in many areas of science and engineering, including robotics [1], haptics [2], computer graphics [3], acoustics [4] and sound synthesis [5]. The

Manuscript received January 0, 0000; revised January 0, 0000.

The research leading to these results has received funding from the EU's Seventh Framework Programme under FET-Open grant agreement $n^{\circ} 222107$ NIW - Natural Interactive Walking, http://www.niwproject.eu.

S. Papetti is with the Dip. Informatica, University of Verona. e-mail: stefano.papetti@univr.it

F. Avanzini is with the Dip. Ingegneria dell'Informazione, University of Padova. e-mail: avanzini@ dei.unipd.it

D. Rocchesso is with the Dip. Arti e Disegno Industriale, IUAV University of Venice. e-mail: roc@iuav.it 
phenomenologically plausible and energy-consistent behavior of contacting bodies is especially crucial in simulations of interactions based on sustained or repeated impacts, such as in rolling [6], scraping, or bouncing [7].

The higher is the upper limit of the perceptual bandwidth (and the rendering rate), the more critical is the accuracy that real-time numerical simulations can afford, thus making the problem of impact modeling increasingly complex when moving from graphic, to haptic, to auditory displays. Whereas for most graphic displays it is sufficient to describe an impact in terms of the ratio between outbound and inbound velocities, in haptic display and in sound synthesis the perceived characteristics of the impact depend on how bodies interact during contact. Therefore, more sophisticated impact models and carefully-designed discretizations are necessary in audio and haptic contexts.

\section{A. Impact Models}

The classic starting point is the Hertz model of collision between two spheres, which can be extended to include internal viscosity [8]. The impact force in such models is the sum of a nonlinear elastic term in the form of a power law of compression - and a dissipative component proportional to the compression velocity - via a second power law of compression. The exponents of the two power laws, as derived for two colliding spheres, are $3 / 2$ and $1 / 2$, respectively [9].

In the context of musical acoustics, Stulov proposed a piano hammer model that includes relaxation properties of felt [10]. Such model has exponents $\alpha$ and $\alpha-1$ for the power laws, and the actual value of $\alpha$ can be used to match experimental data. Other models exist that take plastic deformations into account, thus introducing abrupt direction changes in the force-compression curves at the transition between loading and unloading [11].

Particularly popular is the model by Hunt and Crossley [12], [1], [13], [14], [15], that generalizes the extended Hertz model by considering a variable exponent that accounts for different contact shapes. In this model, the power laws in the elastic and dissipative term are considered to be equal, thus allowing easier closed-form calculations [16]. Despite not being fully justified in physical terms, the Hunt-Crossley model has been quite successful in some areas of engineering because it allows to derive the phase trajectories in closed form, and because it is sufficiently complex to represent a wide variety of contact phenomena. In our work, we adopted this model and extended the range of the available analytical results. 


\section{B. Applications in acoustic modeling}

Contact models can serve as a basis for developing models of acoustic phenomena. In the context of physically-based sound synthesis, the Hunt-Crossley model has been used to develop an impact sound model [5], where a generic resonating object is used in place of the classic rigid wall.

Other models of more complex acoustic phenomena have been developed based on the very same impact model studied here. As an example, a bouncing sound model [7] has been obtained by superimposing a constant force, which simulates gravity, on a plain impact sound model. Also, a rolling sound model [6] has been implemented by driving an impact sound model by means of a physically-inspired control layer. More precisely, the continuous interaction of a ball rolling on a surface has been modeled as a dense temporal sequence of micro-impacts driven by the geometry of the contacting surfaces, and modulated by the ball's asymmetry.

Accurate and consistent impact modeling is crucial in some audio-haptic rendering applications, such as interactive floors or shoes with vibratory and sonic augmentation [17].

In the context of musical sound synthesis, the piano and other percussive musical instruments have also been modeled by using dissipative impact models [10].

\section{Issues with discrete time}

A wide range of numerical methods can be employed to discretize the interaction of impacting bodies. Given a reference continuous-time system, such as the Hunt-Crossley model, the goal is to obtain numerical quantities that follow the continuous-time trajectories as closely as possible, at an affordable computational cost. Thus, efficiency and accuracy are central issues.

Another important goal is energy consistency, especially in the case of repeated or sustained contacts. A numerical method, albeit being accurate, can introduce spurious oscillations or instabilities if it fails in terms of energy conservation. This justifies the development of energy-based methods, i.e. numerical schemes based on the definition of a numerical energy that is proved to be conserved in lossless conditions [18]. These provably-stable discrete-time models can be derived for some continuous-time models, including some nonlinear oscillators, but are not available for more general nonlinear contact models.

As for applications which make use of impact/contact models, energy inconsistencies are a recurring issue. In computer graphics, where the constraint of low frame rates makes numerical systems prone to instabilities [3], a typical example is provided by a steady object in resting contact with a rigid floor: when the system does not retain passivity, the object can move upward and bounce [19]. Similar issues are 
encountered in simulations of haptic contact, where stiffness values are usually limited by requirements on system passivity [20], [21], whereas higher values can cause the system to become unstable, for example oscillating, or reacting actively to the input. In numerical sound synthesis by physical models [22], artifacts and inconsistencies can become audible especially in situations of sustained or repeated contact interactions, as in rolling, sliding or bouncing.

Instead of aiming at provably-stable numerical methods, this study looks at the accuracy of some methods commonly found in physics-based engines [3], and measure their performance in following the theoretical phase trajectories and in reproducing the energy exchanges that occur in the continuous-time impact model.

\section{Outline}

In this paper, the Hunt-Crossley impact model is first characterized by proving some novel analytical results. Such results are then used as a reference to compare the accuracy of several numerical simulations of the model, obtained by discretizing the continuous-time equations with a number of widely used numerical methods. It is shown that for some regions of the parameter space, the trajectories of the discretized systems may significantly drift from the analytically-derived curves. Finally, by exploiting the provided analytical results, two approaches are proposed which allow to improve the accuracy of the numerical simulations, thus restoring their energy consistency.

More in detail, the main contribution of this work can be summarized as follows:

1) Novel approximate closed-form expression for the outbound velocity, and quantification of its relative error. Expression of the total energy (Hamiltonian) as a function of compression velocity (Section $\Pi-\mathrm{A}$ ).

2) Analysis of the distortion caused by a constant external force on the analytically derived phase portraits. This is of some importance for applying the results to real-world simulations (Section [II-B).

3) Comparison of the analytically-derived phase portrait and Hamiltonian with those obtained by applying four relevant numerical methods to the Hunt-Crossley impact model. Two critical cases are examined: low dissipation and hard impact (Section ЩI-B).

4) Proposal of two correction methods for numerical models, one based on the analytic dependence of compression on velocity, and one based on a constraint on the outbound velocity (Section [V]. The remainder of the paper is organized as follows. Section $\Pi$ contains the analytical study, Section $\amalg$ describes different numerical methods which are used for discretizing the continuous-time system, while in Section $\Pi$ II-B the corresponding numerical simulations are compared. Sections IV-A and IV-B show 
how the provided analytical results can be used to improve the behavior of the numerical simulations. Finally, in Sections IV-C and IV-D the computational cost and accuracy of simulations with and without corrections are compared and evaluated.

\section{IMPACT MODEL}

The Hunt-Crossley impact model [12] is described by the following non-linear equation describing the impact force:

$$
f(x, v)= \begin{cases}k x^{\alpha}+\lambda x^{\alpha} v=k x^{\alpha} \cdot(1+\mu v), & x>0 \\ 0 & , \quad x \leq 0\end{cases}
$$

where $x$ is the compression, $v=\dot{x}$ is the compression velocity, $\alpha>1$ is the exponent of a power law and represents the local shape of contact surfaces, $k$ is the stiffness coefficient, and $0 \leq \lambda \leq k$ is the damping coefficient. The mathematically convenient term $\mu(=\lambda / k)$ allows to simplify some closed-form calculations. The impact force model thus represents a non-linear spring of constant $k$ in parallel with a non-linear damper of constant $\lambda$. The term $k x^{\alpha}$ corresponds to the elastic component, while $\lambda x^{\alpha} v$ represents the dissipation due to internal friction.

Marhefka and Orin [1] made use of the Hunt-Crossley model in order to represent the impact between a lumped point-mass and a rigid wall (representing a comparatively massive surface which does not move during collision), therefore considering the system described by the equation:

$$
m a(t)=-f(x(t), v(t))
$$

where $m$ is the mass, and $a$ is its acceleration. In this basic case, during contact the compression and the compression velocity are respectively equivalent to the displacement and the velocity of the point-mass.

\section{A. Properties and analytical results}

Thanks to the simple form of (2), the model can be treated analytically and some of its properties can be inferred. Hereafter the initial conditions $x(0)=0$ and $\dot{x}(0)=v_{\text {in }}$ are considered, that is to say that the point-mass hits the rigid wall with velocity $v_{\text {in }}$ at time $t=0$.

1) Compression: It is shown in [1] that from (2) it follows:

$$
x(v)=\left[\frac{m(\alpha+1)}{k \mu^{2}} \cdot\left(-\mu\left(v-v_{\text {in }}\right)+\log \left|\frac{1+\mu v}{1+\mu v_{\text {in }}}\right|\right)\right]^{\frac{1}{\alpha+1}}
$$

which can be exploited for plotting the phase portraits on the $(x, v)$ plane shown in Fig. 11 From Fig. 1 it can be inferred that, due to the viscous dissipation occurring during contact, the relation $v(t+d t)<v(t)$ holds, and in particular the output velocity $v_{\text {out }}$ is always smaller in magnitude than the corresponding 


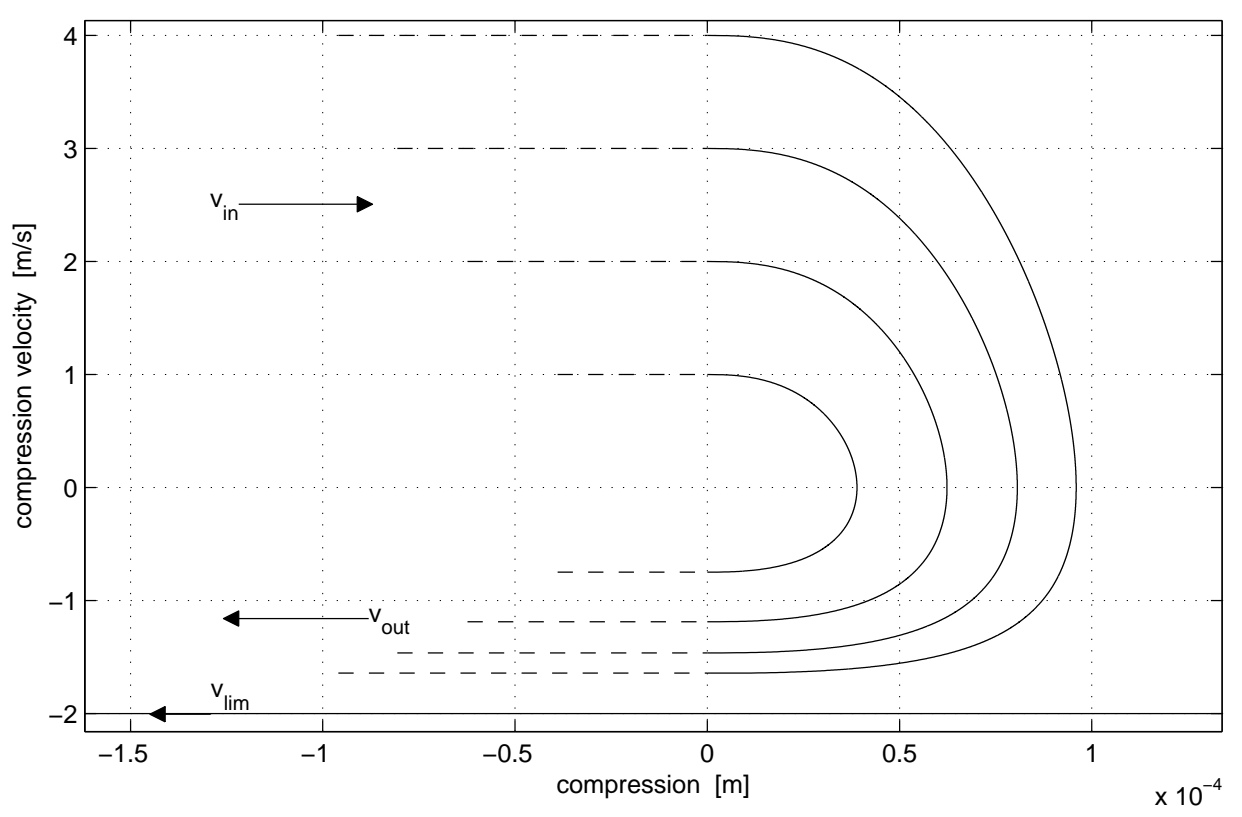

Fig. 1. Phase portraits for varying input velocities: $v_{\text {in }}=1 \ldots 4 \mathrm{~m} / \mathrm{s}$. Other values of parameters are: $m=10^{-2} \mathrm{~kg}$, $k=10^{9} \mathrm{~N} / \mathrm{m}^{\alpha}, \mu=0.5 \mathrm{~s} / \mathrm{m}, \alpha=1.5$. Solid lines represent the mass trajectory during contact; dashed lines represent free motion.

$v_{\text {in. }}$. Moreover, for increasing $v_{\text {in }}$ 's, $v_{\text {out }}$ converges to the limit value $v_{\lim } \triangleq-1 / \mu$. The line $v=v_{\lim }$ represents the trajectory where the elastic and dissipative terms cancel, and separates two regions of the phase space, each of which is never entered by trajectories started in the other one.

Equation (3) allows to infer the maximum compression experienced during contact, which occurs when the compression velocity equals zero:

$$
x_{\max }=x(0)=\left[\frac{m(\alpha+1)}{k \mu^{2}} \cdot\left(\mu v_{\text {in }}+\log \left|\frac{1}{1+\mu v_{\text {in }}}\right|\right)\right]^{\frac{1}{\alpha+1}} .
$$

As remarked in [1], (1) together with Fig. 1] show that the force $f$ becomes sticky (inward) when $v<v_{\text {lim. }}$. However there is no physical inconsistency in this "stickiness" property, and indeed this never occurs for trajectories with initial conditions $x(0)=0$ and $\dot{x}(0)=v_{\text {in }}>0$.

Finally, by substituting (3) in (2) the compression-force characteristics during collision can be plotted, which are shown in Fig. 2. It can be noted that the dissipative term $\lambda x^{\alpha} v$ introduces hysteresis around the curve $k x^{\alpha}$.

2) Output velocity: The restitution coefficient $E$ is defined as:

$$
E \triangleq\left|\frac{v_{\text {out }}}{v_{\text {in }}}\right| .
$$




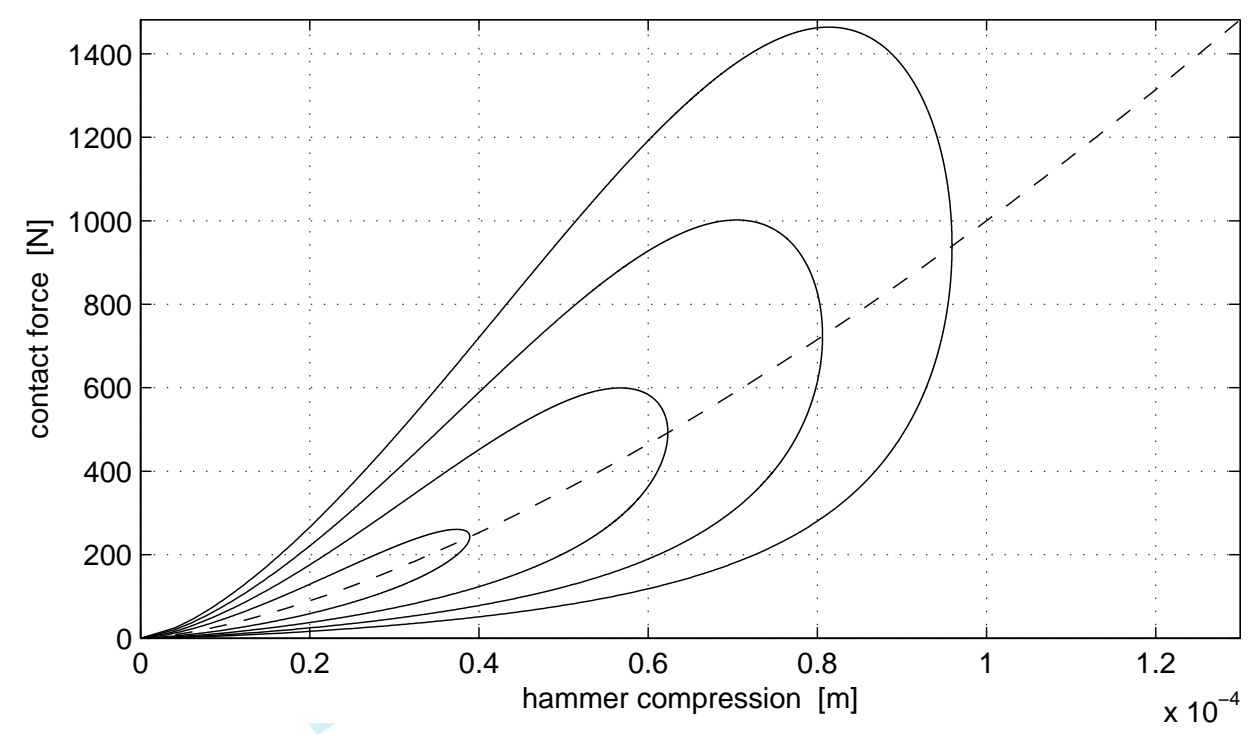

Fig. 2. Compression-force characteristics for varying input velocities: $v_{\text {in }}=1 \ldots 4 \mathrm{~m} / \mathrm{s}$. Solid lines represent the case when dissipation is taken into account (the values of parameters are the same as in Fig. 1). The dashed line represents the curve $k x^{\alpha}$, where no dissipation is considered $(\lambda=0)$.

Note that $v_{\text {in }}$ and $v_{\text {out }}$ correspond to the roots of the right-hand side of (3), that is the points where $x=0$. As a result, $v_{\text {out }}$ can be defined implicitly from (3) as a function of $\left(\mu, v_{\text {in }}\right)$ only:

$$
\mu v_{\text {out }}-\log \left|1+\mu v_{\text {out }}\right|=\mu v_{\text {in }}-\log \left|1+\mu v_{\text {in }}\right|
$$

This implies that $\mu v_{\text {out }}$ is a function of $\mu v_{\text {in }}$ only, and therefore $E$ is also a function of $\mu v_{\text {in }}$ only.

Analytical derivations of the dependence $E\left(\mu v_{\text {in }}\right)$ have been classically performed in the limit of small initial velocities and/or small dissipation [12]1 1 However, we suggest that a non-local approximation $\tilde{v}_{\text {out }}$ can be empirically determined as an ansatz which fits the curve $E\left(\mu v_{\text {in }}\right)$ in the two limit regions $\mu v_{\text {in }} \rightarrow 0_{+}$and $\mu v_{\text {in }} \rightarrow+\infty$, thus obtaining 2 :

$$
\tilde{v}_{\text {out }}\left(\mu, v_{\text {in }}\right)=v_{\lim }\left[1-\left(\sum_{j=0}^{n} b_{j} \cdot v_{\text {in }}^{j}\right) e^{-2 \mu v_{\text {in }}}\right]
$$

where, in the case $n=4$, the coefficients $b_{j}$ are:

$$
b_{0}=1, \quad b_{1}=\mu, \quad b_{2}=\frac{2}{3} \mu^{2}, \quad b_{3}=\frac{2}{9} \mu^{3}, \quad b_{4}=\frac{14}{135} \mu^{4} .
$$

\footnotetext{
${ }^{1}$ Appendix $\mathrm{A}$ provides an example.

${ }^{2}$ See Appendix B for details.
} 


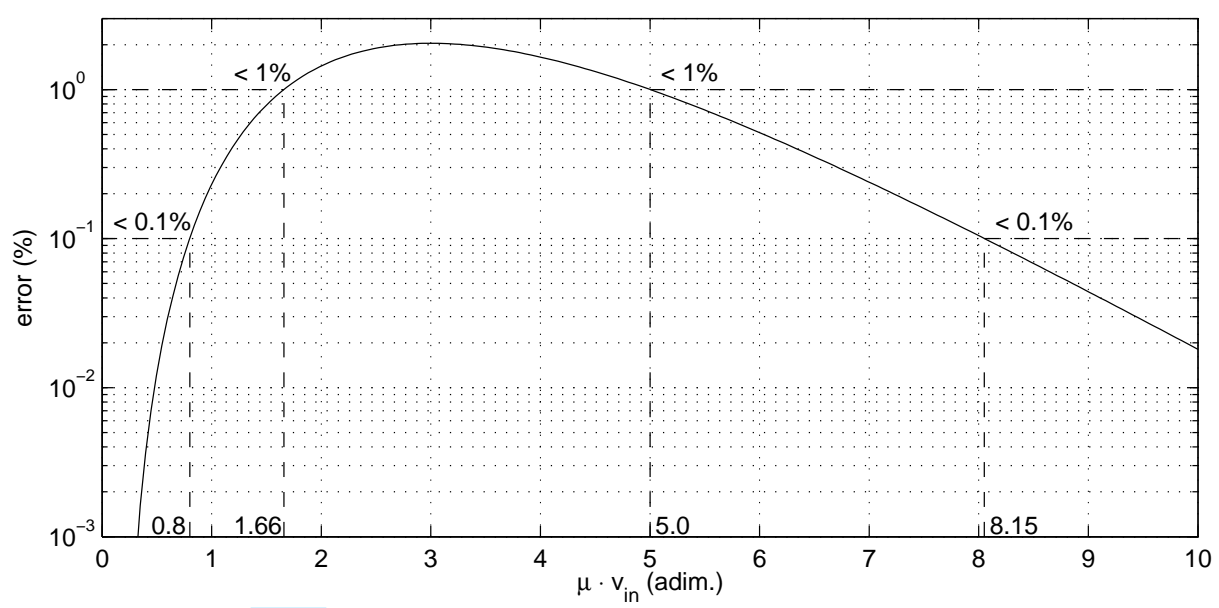

Fig. 3. Log-scaled percentage error of the output velocity approximated by $\tilde{v}_{\text {out }}$, with respect to the value computed numerically as a zero of (6). Ranges of $\mu v_{\text {in }}$ are shown, for which the maximum error is respectively less than $1 \%$ and $0.1 \%$.

From now on, unless specified otherwise, the notation $\tilde{v}_{\text {out }}$ will refer to the fourth-order approximation provided by (7) and the coefficients (8). Conventional iterative zero-finding methods applied to (6) can always be used to compute a more precise release velocity at a higher computational cost (see IV-C).

Fig. 3 shows the error introduced by the approximate value $\tilde{v}_{\text {out }}$, when compared to the corresponding value computed numerically as a zero of (6).

3) Contact time: It is shown in [23] that the contact time can be expressed as:

$$
\begin{aligned}
& \tau=\left(\frac{m}{k}\right)^{\frac{1}{\alpha+1}} \cdot\left(\frac{\mu^{2}}{\alpha+1}\right)^{\frac{\alpha}{\alpha+1}} \cdot \\
& \cdot \int_{v_{\text {out }}}^{v_{\text {in }}} \frac{1}{(1+\mu v)\left[-\mu\left(v-v_{\text {in }}\right)+\log \left|\frac{1+\mu v}{1+\mu v_{\text {in }}}\right|\right]^{\frac{\alpha}{\alpha+1}}} .
\end{aligned}
$$

Equation (9) states that the contact time $\tau$ depends only on $\mu$, the exponent $\alpha$ and the ratio $m / k$, plus obviously the impact velocity $v_{\text {in }}$. Since neither $m$ nor $k$ affect the value of the integral (recall that $v_{\text {out }}$ depends only on $\mu$ and $v_{\text {in }}$ ), it follows that, given a fixed $v_{\text {in }}$, the proportionality $\tau \sim(m / k)^{1 /(\alpha+1)}$ holds.

From an auditory point of view the value of the contact time is strongly correlated to the perceived "hardness" of the impact [23], [24]. Namely, as the contact time decreases, the perceived hardness increases. Recalling the power-law dependence above and (1) it follows that, for a fixed mass $m$, "hard" and "soft" impacts correspond respectively to high and low force values. 
4) Energy properties and behavior: The energy variation in a mechanical system can be calculated as the work made by the non-conservative forces $f_{\text {nc }}$ acting on the system along a certain path $x_{1} \rightarrow x_{2}$ :

$$
\Delta H=\int_{x_{1}}^{x_{2}} f_{\mathrm{nc}}(x) d x=\int_{t_{1}}^{t_{2}} f_{\mathrm{nc}}(t) v(t) d t=-\Delta \Lambda
$$

where $H$ is the total energy content, known as the Hamiltonian, $\Lambda$ is the energy dissipation, and the second integral is obtained by considering that $t_{1}$ and $t_{2}$ correspond respectively to the instants when the displacements $x_{1}$ and $x_{2}$ are reached. The Hamiltonian $H$ is the sum of potential and kinetic energies, hereafter named $V$ and $T$, respectively:

$$
H(t)=V(t)+T(t)
$$

With regard to the system represented by (2), $T$ is related to the dynamics of the point-mass, which is described by the left-hand side of (2), while $V$ is related to the elastic component of the impact force of (1).

In agreement with the last integral in (10), multiplying both sides of (2) by $v(t)=d x / d t$ and timeintegrating them, gives:

$$
\begin{aligned}
& \underbrace{\int_{0}^{t} m a(s) v(s) d s}_{T(t)-T_{0}}= \\
& =-\underbrace{\int_{0}^{t} k x(s)^{\alpha} v(s) d s}_{V(t)}-\underbrace{\int_{0}^{t} \lambda x(s)^{\alpha} v(s)^{2} d s}_{\Lambda(t)>0}
\end{aligned}
$$

where the force expression of (1) has been considered in the case $x>0$ only. The first two integrals in (12) can be solved explicitly, obtaining:

$$
V(t)=\frac{k x(t)^{\alpha+1}}{\alpha+1}, \quad T(t)=\frac{m v(t)^{2}}{2} .
$$

Considering a system where the point-mass travels with velocity $v_{\text {in }}$ before an impact occurs, then the initial Hamiltonian corresponds to the initial kinetic energy:

$$
H_{0}=T_{0}=\frac{m v_{\text {in }}^{2}}{2} .
$$

From (10) it follows that at each time instant $t$ :

$$
H(t)=H_{0}-\Lambda(t)
$$

and, since $\Lambda(t)>0$, the following inequalities hold:

$$
0 \leq H(t+d t) \leq H(t) .
$$




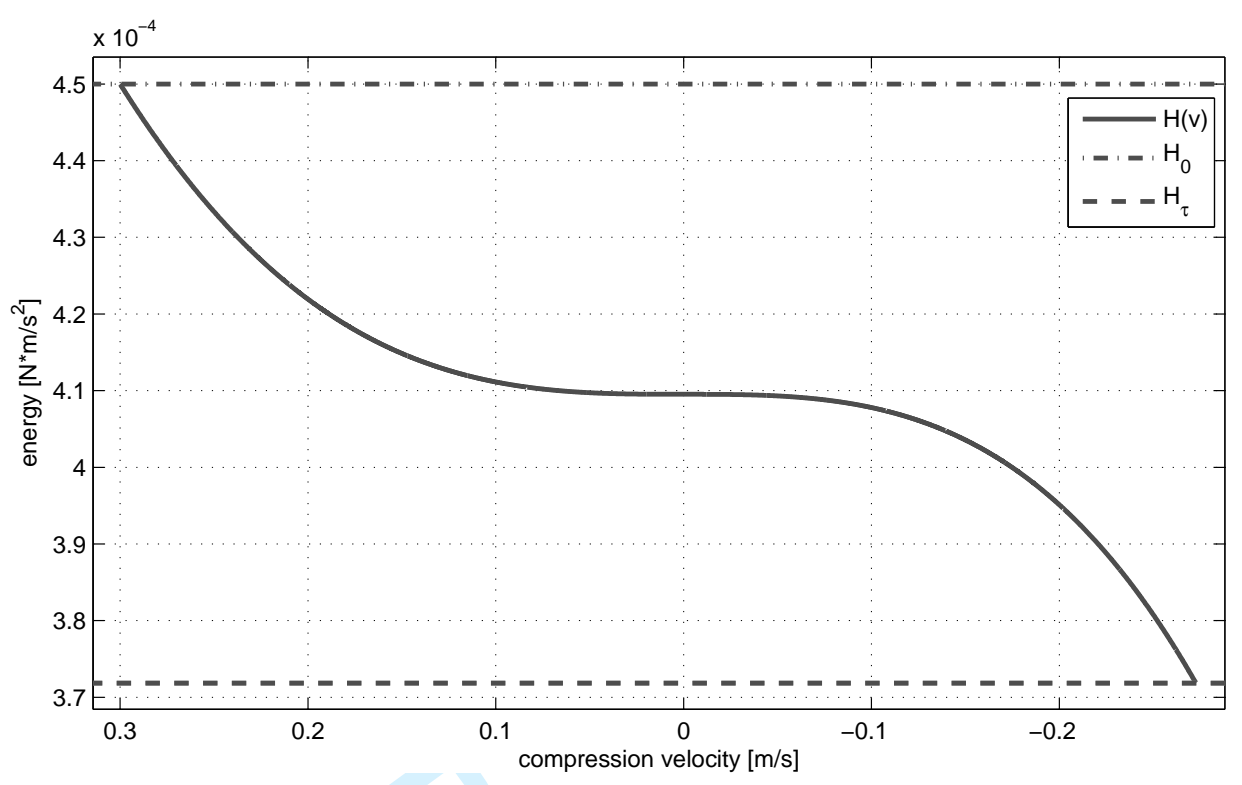

Fig. 4. Compression velocity-Hamiltonian characteristic. The two horizontal lines display $H_{0}=T_{0}$ and $H_{\tau}=T_{\tau}$, that is respectively the initial and the final Hamiltonian. The values of parameters are the same as in Fig. 1 for $v_{\text {in }}=1 \mathrm{~m} / \mathrm{s}$. The compression velocity axis has been inverted, thus allowing to read the graph from left to right.

Indicating $\tau$ as the instant when an impact ends, the final Hamiltonian of the system, that is the energy content right after contact, can be written as:

$$
H_{\tau}=T_{\tau}=\frac{m v_{\mathrm{out}}^{2}}{2}
$$

Also, the total amount of energy dissipation occurred during contact is:

$$
\Delta H_{\tau}=H_{\tau}-H_{0}=-\Lambda_{\tau}
$$

which corresponds to the area enclosed by the hysteresis loops shown in Fig. 2

As for the rightmost integral in (12), which is non-solvable, an equivalent expression can be obtained by substituting the complementary results for the remaining integrals:

$$
\Lambda(t)=\int_{0}^{t} \lambda x(s)^{\alpha} v(s)^{2} d s=\frac{m\left(v_{\mathrm{in}}^{2}-v(t)^{2}\right)}{2}-\frac{k x(t)^{\alpha+1}}{\alpha+1} .
$$

Finally, by substituting (3) in (19) and recalling (15), the following expression in $v$ only is found:

$$
H(v)=H_{0}-\Lambda(v)=\frac{m}{2} v^{2}-\frac{m}{\mu}\left(v-v_{\text {in }}\right)+\frac{m}{\mu^{2}} \log \left|\frac{1+\mu v}{1+\mu v_{\text {in }}}\right|
$$

which can be used for plotting the curve shown in Fig. 4 


\section{B. Addition of a constant external force}

When a constant external force $f_{e}$ (e.g. the force of gravity) is applied to the point mass, (2) has to be rewritten as:

$$
m a(t)+f_{e}=-f(x(t), v(t)) .
$$

Unfortunately, in this case no closed-form analytical results can be found as those described in In more detail, multiplying both sides of (21) by $v(t)$ and time-integrating them, an equation is found where an unsolvable integral is present, this way preventing to directly obtain explicit-form expressions for $x(v), \tilde{v}_{\text {out }}$ and $\tau$.

Rewriting the 2nd-order equation (21) as a system of 1st-order equations:

$$
\left\{\begin{array}{l}
\dot{x}=v \\
\dot{v}=-\frac{k}{m} x^{\alpha}-\frac{\lambda}{m} x^{\alpha} v-\frac{f_{e}}{m}
\end{array}\right.
$$

the equilibrium point of the system is found to be $\left(x_{\mathrm{eq}}, v_{\mathrm{eq}}\right)=\left(-f_{e}^{1 / \alpha} / k, 0\right)$, which corresponds to the compression offset in stationary conditions.

As Fig. 5 shows, for positive values of $f_{e}$ and $v_{\text {in }}$, the velocity of the point mass during the compression phase is generally greater than in the case when $f_{e}=0$. In particular, at the beginning of contact interaction, since $f_{e}$ is higher than the current impact force $f$, the compression velocity exceeds $v_{\text {in }}$. On the other hand, compared to the case when $f_{e}=0$, during the decompression phase the absolute value of the point mass velocity $v$ decreases, resulting in lower output velocities. Moreover, the resulting maximum compression is always greater than that calculated by (4).

\section{NUMERICAL SIMULATIONS}

In this section, the continuous-time system described by (2) is discretized by means of several numerical methods, and the resulting numerical systems are studied.

\section{A. Numerical methods}

Different numerical methods were considered, which are commonly used in various fields of applications spacing from computer graphics, to physical simulation of dynamical systems and digital signal processing. Thanks to their low order - which generally results in low computational cost - the chosen methods are particularly suitable for real-time applications:

- The trapezoid rule is popular to translate analog filter structures to discrete-time filters, and it is the basis for wave digital filters; 


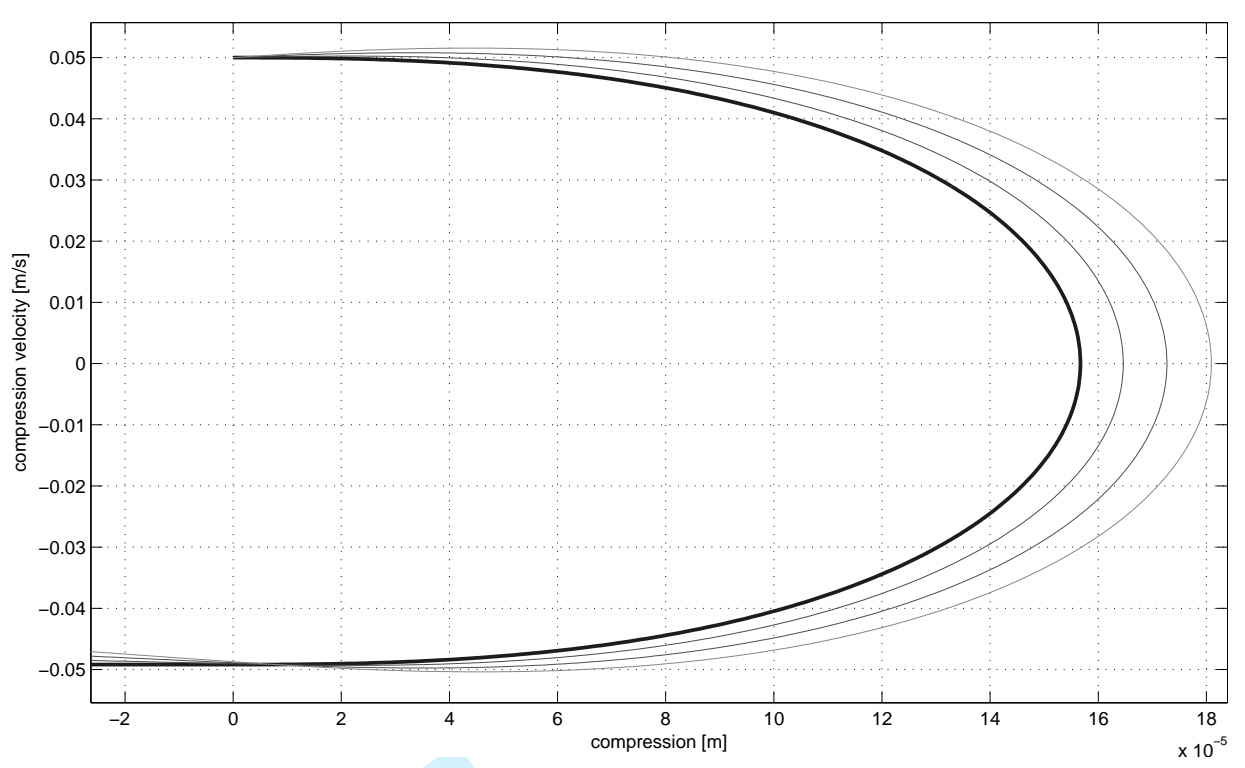

Fig. 5. Phase portraits of impacts for different external forces applied: $f_{e}=m \cdot(1,2,3) \mathrm{N}$. The bold line represents the case where $f_{e}=0$. The values of parameters are the same as in Fig. 1 except for $k=10^{5} \mathrm{~N} / \mathrm{m}^{\alpha}$ and $v_{\text {in }}=0.05 \mathrm{~m} / \mathrm{s}$. Notice that, due to the lack of analytical results when an external force is present, the phase portraits have been obtained as the result of numerical simulations.

- Verlet integration is popular in physics-based graphic engines;

- Heun is a 2nd-order method (complexity similar to the previous two) representative of the RungeKutta family;

- 4th-order Runge-Kutta is expected to be more accurate, and more expensive.

Following the standard notation in numerical analysis, the integration step is a constant named $h(=$ $\left.1 / F_{s}\right)$.

1) 1-step Adams-Moulton (AM1): is a A-stable 2nd-order implicit method [25], also known as bilinear transformation, or trapezoid rule.

Discretizing (2) results in the following equation in state-space form:

$$
\left[\begin{array}{l}
x_{n+1} \\
v_{n+1}
\end{array}\right]=\left[\begin{array}{ll}
1 & h \\
0 & 1
\end{array}\right]\left[\begin{array}{l}
x_{n} \\
v_{n}
\end{array}\right]+\left[\begin{array}{c}
\frac{h^{2}}{4 m} \\
\frac{h}{2 m}
\end{array}\right]\left[f_{n+1}+f_{n}\right]
$$

where the expression for the discrete-time force is obtained by replacing the continuous-time variables $x(t)$ and $v(t)$ in (1) with their discrete-time counterparts.

Since the AM1 method is implicit, (23) is also in implicit form, and this is reflected in the instantaneous relationship between $\left[\begin{array}{ll}x_{n+1} & v_{n+1}\end{array}\right]^{T}$ and $f_{n+1}$. Unfortunately, since $f_{n+1}$ also has an instantaneous 
dependence on $x_{n+1}$ and $v_{n+1}$ (given by (10), the discrete-time counterpart of the system described by (2) contains a delay-free loop, which is not directly computable and - because of the nonlinear dependence $f(x, v)$ - needs some special handling in order to be solved. In particular, the K-method [26] together with Newton's method [25] are used, weighing on the efficiency of the simulation (see IV-C).

2) Verlet: is a 2nd-order explicit method commonly used in computer graphics [3], video games, and molecular dynamics simulation, where it is typically used for integrating Newton's equation of motion in order to describe the trajectory of moving particles. The one used here is a variant, called velocity Verlet, which provides better handling of the velocity variable and can be seen as a predictor-corrector method.

Discretizing (2), results in the following implementation scheme:

$$
\begin{aligned}
& x_{n+1}=x_{n}+h v_{n}+\frac{h^{2}}{2} \frac{f_{n}}{m}, \\
& v_{n+\frac{1}{2}}=v_{n}+\frac{h}{2} \frac{f_{n}}{m}, \quad \text { predictor, } \\
& f_{n+1}=f\left(x_{n+1}, v_{n+\frac{1}{2}}\right), \\
& v_{n+1}=v_{n+\frac{1}{2}}+\frac{h}{2} \frac{f_{n+1}}{m}, \quad \text { corrector. }
\end{aligned}
$$

It should be noted that this algorithm assumes that $f_{n+1}$ only depends on the predicted velocity $v_{n+\frac{1}{2}}$, which clearly gives rise to inaccuracies.

3) Heun: is a predictor-corrector explicit method [25], [3], with the forward Euler method as predictor and the trapezoid rule as corrector. It can also be seen as a 2nd-order Runge-Kutta method (RK2).

Discretizing (2) results in the following implementation scheme:

$$
\begin{aligned}
\tilde{v}_{n+1} & =v_{n}+h \frac{f_{n}}{m}, \quad \text { predictor, } \\
x_{n+1} & =x_{n}+\frac{h}{2}\left(v_{n}+\tilde{v}_{n+1}\right), \\
f_{n+1} & =f\left(x_{n+1}, \tilde{v}_{n+1}\right), \\
v_{n+1} & =v_{n}+\frac{h}{2} \frac{f_{n}+f_{n+1}}{m}, \quad \text { corrector. }
\end{aligned}
$$

Again, it should be noted that both $x_{n+1}$ and $f_{n+1}$ only depend on the predicted velocity $\tilde{v}_{n+1}$, and this gives rise to inaccuracies.

4) 4th-order Runge-Kutta (RK4): is an explicit iterative method [25], [3] which is widely used to solve ODEs with improved accuracy. 
Discretizing (2) results in the following implementation scheme:

$$
\begin{aligned}
& x_{n+1}=x_{n}+\frac{1}{6}\left(l_{1}+2 l_{2}+2 l_{3}+l_{4}\right), \\
& v_{n+1}=v_{n}+\frac{1}{6}\left(k_{1}+2 k_{2}+2 k_{3}+k_{4}\right)
\end{aligned}
$$

where:

$$
\begin{gathered}
l_{1}=h v_{n}, \quad l_{2}=h\left(v_{n}+\frac{k_{1}}{2}\right), \\
l_{3}=h\left(v_{n}+\frac{k_{2}}{2}\right), \quad l_{4}=h\left(v_{n}+k_{3}\right), \\
k_{1}=h \frac{f_{n}}{m}, \quad k_{2}=h \frac{f\left(x_{n}+\frac{l_{1}}{2}, v_{n}+\frac{k_{1}}{2}\right)}{m}, \\
k_{3}=h \frac{f\left(x_{n}+\frac{l_{2}}{2}, v_{n}+\frac{k_{2}}{2}\right)}{m}, \quad k_{4}=h \frac{f\left(x_{n}+l_{3}, v_{n}+k_{3}\right)}{m} .
\end{gathered}
$$

It should be noted that, for each sample, both the velocity and the non-linear force of (1) need to be evaluated four times, therefore strongly affecting the efficiency of the simulation (see IV-C).

\section{B. Experimental results}

In order to evaluate the chosen numerical methods, it is useful to compare the behavior of the corresponding simulations against the known analytical results (see $\amalg-\mathrm{A}$ ).

The main references used to quantitatively assess the reliability of a particular numerical method during contact are equations (3) and (20), which express respectively the compression $x$ and the Hamiltonian $H$ as functions of the compression velocity $v$. The errors on $x$ and $H$ are then measured as the maximum deviations 3 of their discrete-time versions from the respective analytical curves $x(v)$ and $H(v)$, in relation to the entire variation range of the quantities considered (i.e. $x_{\max }$ and $\Delta H_{\tau}$ ). In detail, taking into account (20) and defining $H^{\operatorname{sim}}\left(x_{n}, v_{n}\right)$ as the Hamiltonian computed using the values of compression and velocity resulting from a numerical simulation 4 and $\Delta H_{\tau}$ as in (18), the maximum deviation on $H$ is calculated by means of the following expression:

$$
\% \operatorname{dev}(H)=100 \cdot\left|\frac{\max _{n}\left\{H^{\operatorname{sim}}\left(x_{n}, v_{n}\right)-H\left(v_{n}\right)\right\}}{\Delta H_{\tau}}\right| .
$$

\footnotetext{
${ }^{3}$ Such measures definitely have a different meaning compared to ordinary relative errors, since the latter would only account for local deviations from the analytical curves. On the other hand, the proposed measure of error is obtained normalizing the absolute error according to the range of variation of the quantities being evaluated, and picking the maximum deviation along such range.

${ }^{4}$ That is, substituting $x_{n}$ and $v_{n}$ in [13]. Not to be confused with the discrete Hamiltonian defined in [22] for finite-difference schemes.
} 
Considering (3) and defining $x_{\max }$ as in (4), the maximum deviation on $x$ is calculated as:

$$
\% \operatorname{dev}(x)=100 \cdot\left|\frac{\max _{n}\left\{x_{n}-x\left(v_{n}\right)\right\}}{x_{\max }}\right| .
$$

Finally, another indicator which allows to evaluate the accuracy and consistency of the simulations at release from contact is provided by the output velocity computed numerically as a zero of (6), which is used to calculate the relative error $\% \operatorname{err}\left(v_{\text {out }}\right)$ of its simulated counterpart $v_{\text {out }}^{\text {sim }}$.

Throughout the following example simulations some values of parameters are kept constant: $m=$ $10^{-2} \mathrm{Kg}$, and $F_{s}=44.1 \mathrm{kHz}$ (i.e. a standard audio sample rate).

1) Non-critical simulations: In order to verify the numerical implementations, the parameters of the model are set to a "safe" configuration, that is to far-from-extreme values. In this case, contact extends over many samples, thus ensuring that the simulations are influenced only to a negligible extent by the chosen sampling rate (see [-Cl) and should more likely behave as the original continuous-time system.

This is confirmed qualitatively by Fig. 6, where the plots of all such simulations substantially overlap and coincide with the analytical curves. Moreover, Table I offers a quantitative evaluation of the simulations, showing the errors introduced by the different numerical methods considered.

\section{TABLE I}

SUMMARY OF ERRORS IN NON-CRITICAL SIMULATIONS. THE VALUES OF PARAMETERS ARE $k=10^{3} \mathrm{~N} / \mathrm{M}^{\alpha}, \mu=0.5 \mathrm{~S} / \mathrm{M}$,

$$
\alpha=1.5, v_{\mathrm{IN}}=0.5 \mathrm{M} / \mathrm{s} .
$$

\begin{tabular}{|l||c|c|c|c|}
\hline errors & AM1 & Verlet & Heun & RK4 \\
\hline$\% \operatorname{dev}(x)$ & 0.255 & 0.018 & 0.319 & 0.005 \\
$\% \operatorname{err}\left(v_{\text {out }}\right)$ & $+2 \cdot 10^{-5}$ & $+2 \cdot 10^{-6}$ & $-3 \cdot 10^{-5}$ & $+2 \cdot 10^{-6}$ \\
$\% \operatorname{dev}(H)$ & $3 \cdot 10^{-4}$ & 0.052 & $4 \cdot 10^{-4}$ & $1 \cdot 10^{-5}$ \\
\hline
\end{tabular}

2) Critical parameter regions: It has been found empirically that when $\tau^{\mathrm{sim}} \leq 4$ samples, the errors on $x, H$ and $v_{\text {out }}$ heavily increase, resulting in an extremely poor reliability of all the simulations. The obvious reason for this behavior is that, since only very few samples of data are available, the numerical systems are totally unable to describe the original continuous-time counterpart. Hence, in the study hereafter, only values of parameters resulting in $\tau^{\text {sim }}>4$ samples are considered.

It has been observed that when $\mu \rightarrow 0_{+}$and/or as the contact time $\tau^{\text {sim }}$ decreases (i.e. for "hard" impacts), the behavior of most numerical implementations tends to become inconsistent with the continuoustime system. Hereafter, the numerical systems are studied for these two critical configurations, respectively 


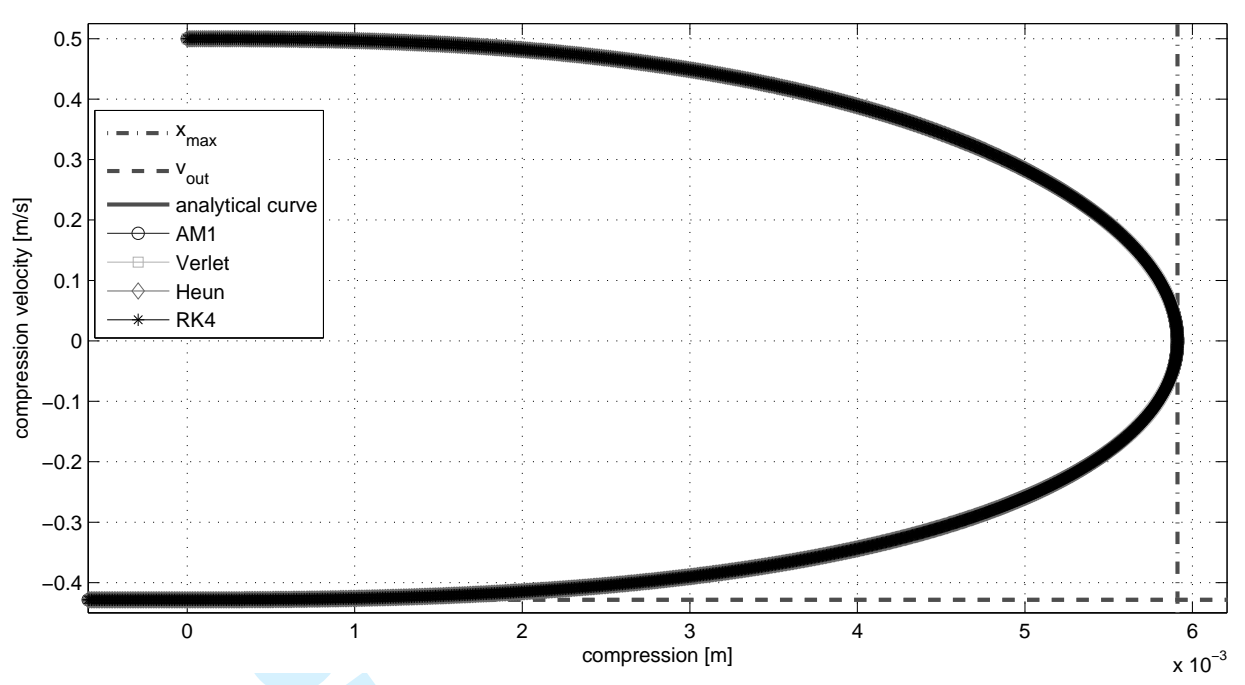

(a) Phase portraits. The two tangent lines represent the maximum compression $x_{\max }$ calculated by (4) and the output velocity $v_{\text {out }}$ computed numerically as a zero of (6).

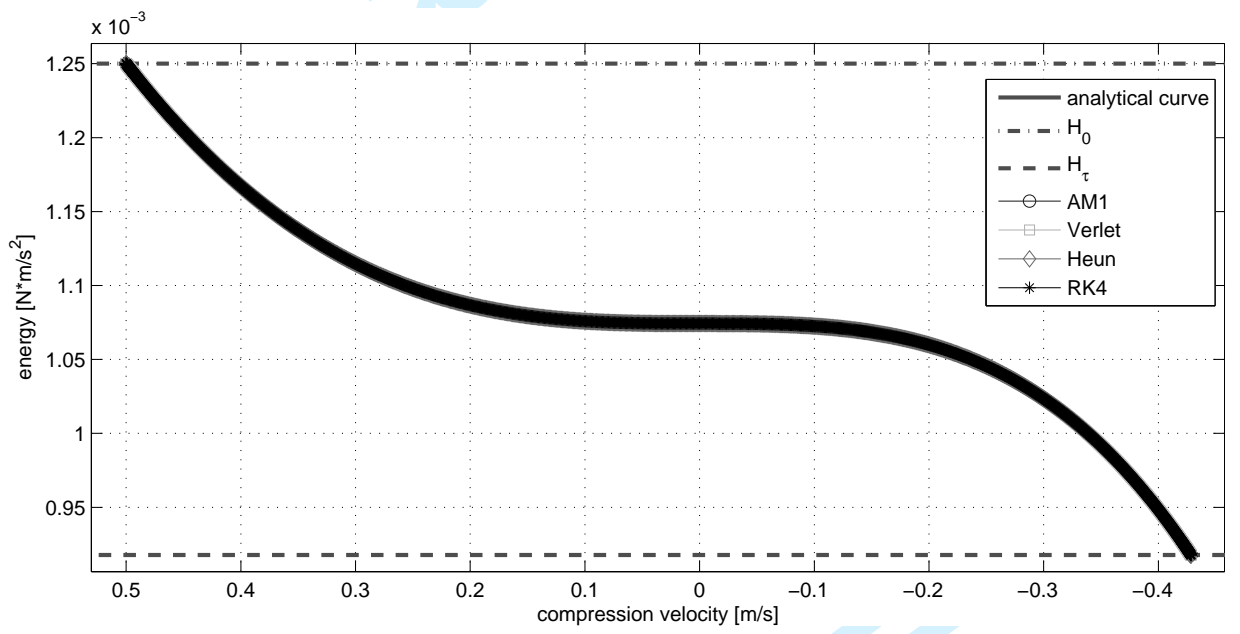

(b) Energy behaviors. The two horizontal lines display $H_{0}=T_{0}$ and $H_{\tau}=T_{\tau}$, i.e. respectively the initial and the final Hamiltonian.

Fig. 6. Qualitative comparison of different methods in non-critical simulations. The values of parameters are $k=10^{3} \mathrm{~N} / \mathrm{m}^{\alpha}$, $\mu=0.5 \mathrm{~s} / \mathrm{m}, \alpha=1.5, v_{\text {in }}=0.5 \mathrm{~m} / \mathrm{s}$.

named case 1 and case 2.

Case 1: low dissipation $\left(\mu \rightarrow 0_{+}\right)$

In case of low dissipation, the Hamiltonian of both Verlet- and Heun-discretized systems is prone to

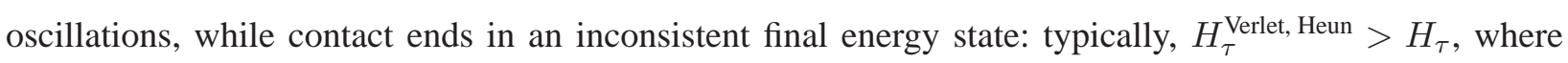




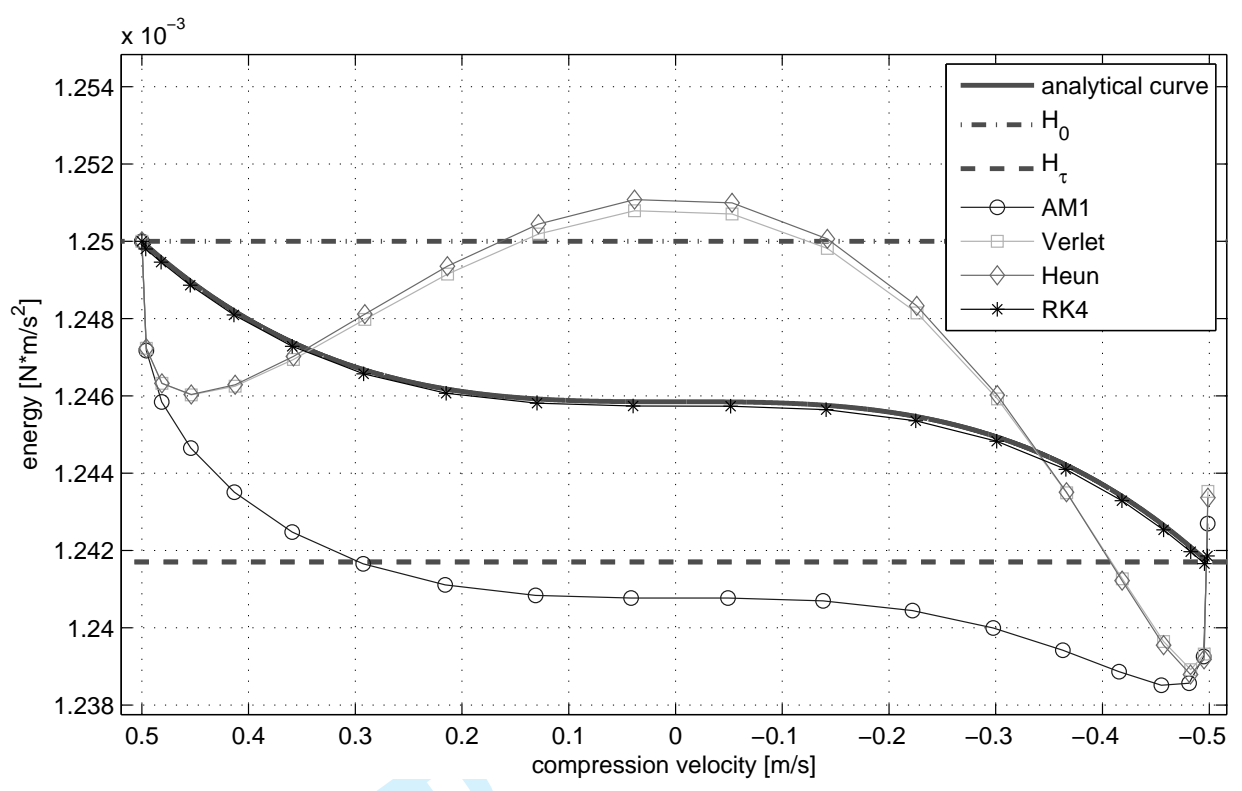

Fig. 7. Comparison of Hamiltonians for different implementations of a simulation example following case 1. The values of parameters are $k=10^{7} \mathrm{~N} / \mathrm{m}^{\alpha}, \mu=0.01 \mathrm{~s} / \mathrm{m}, \alpha=1.3, v_{\text {in }}=0.5 \mathrm{~m} / \mathrm{s}$. The contact time equals 19 samples.

$H_{\tau}$ is defined as in (17) and is calculated using values of $v_{\text {out }}$ computed numerically as zeros of (6). As for AM1-discretized systems, these generally tend to dissipate too much energy during contact (i.e. $H^{\mathrm{AM} 1}<H$ ), while slightly gaining spurious energy as the contact ends (i.e. $H_{\tau}^{\mathrm{AM} 1}>H_{\tau}$ ). On the other hand, RK4-discretized systems generally behave quite consistently both during and after the contact interaction (i.e. $H^{\mathrm{RK} 4} \approx H$ ).

Fig. 7 7 shows the Hamiltonian of a simulation example with low dissipation $(\mu=0.01)$, while Table $\llbracket(a)$ shows the resulting errors.

Case 2: hard impacts

With the exception of RK4-discretized systems, in this case the simulations usually show more spread errors: $x^{\text {sim }}, H^{\text {sim }}$ and $v_{\text {out }}^{\text {sim }}$ tend to substantially deviate from the respective analytical results.

Fig. 8 shows a hard impact simulation example following the values of parameters adopted in Fig. 1 for $v_{i n}=1 \mathrm{~m} / \mathrm{s}$, while Table 【[(b) shows the corresponding errors. The resulting contact time $\tau$ equals 6 samples.

It is worth noticing that the RK4 method has been proved to behave quite consistently across disparate configurations of parameters. Therefore, a highly oversampled RK-discretized system can be taken as a 


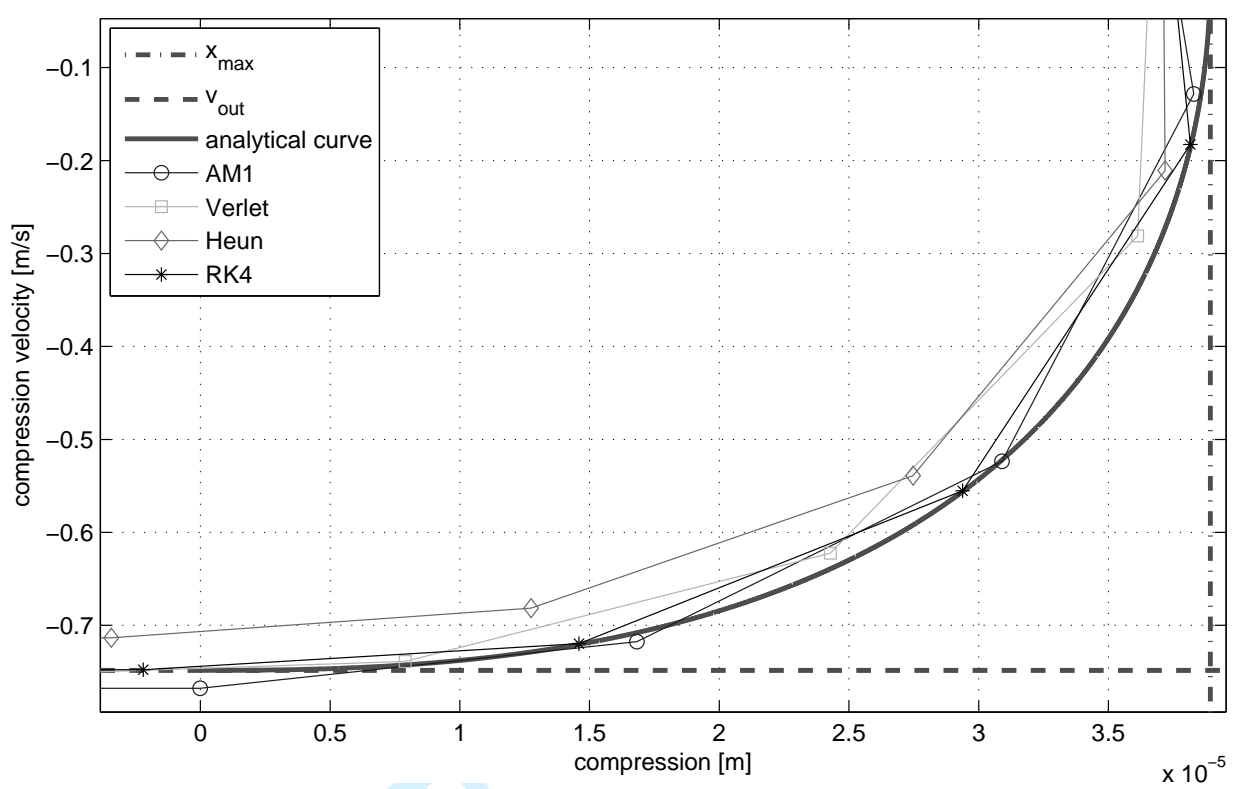

(a) Detail of phase portraits.

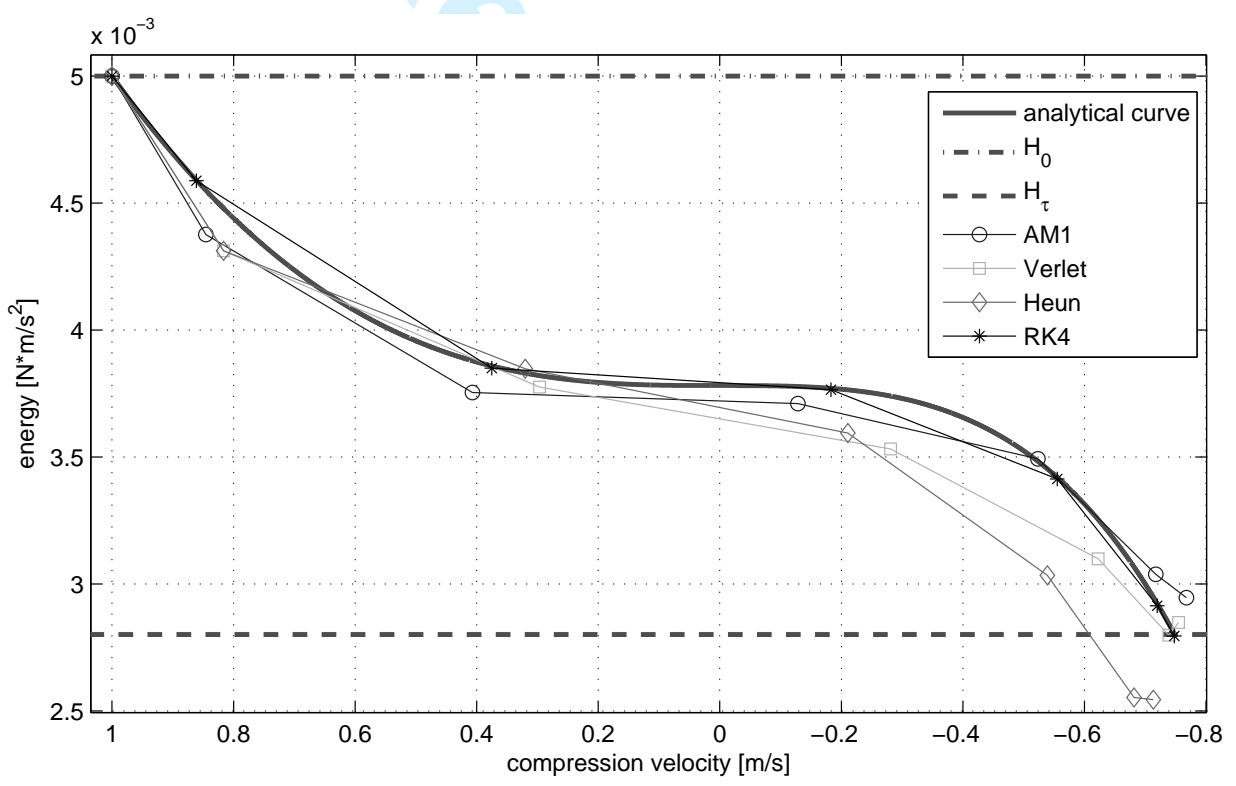

(b) Hamiltonians.

Fig. 8. Comparison of phase portraits and Hamiltonians for different implementations of a simulation example following case 2. The values of parameters are the same as in Fig. 1 with $v_{\text {in }}=1 \mathrm{~m} / \mathrm{s}$. The contact time equals 6 samples.

reference, able to provide extremely accurate simulations. 
TABLE II

SUMMARY OF ERRORS IN EXAMPLE SIMULATIONS OF case 1 AND case 2. THE LAST COLUMN SHOWS THE ERROR YIELDED BY THE APPROXIMATE VALUE $\tilde{v}_{\text {OUT }}$ WITH RESPECT TO THE VALUE COMPUTED NUMERICALLY: IT IS WORTH NOTICING THAT IN BOTH CASES THE ERROR IS LOWER THAN THOSE YIELDED BY THE SIMULATIONS.

(a) Simulation example following case 1. The values of parameters are the same as in Fig. 7

\begin{tabular}{|l||c|c|c|c|c|}
\hline errors & AM1 & Verlet & Heun & RK4 & $\tilde{v}_{\text {out }}$ \\
\hline$\% \operatorname{dev}(x)$ & 1.011 & 1.083 & 1.136 & 0.052 & - \\
$\% \operatorname{err}\left(v_{\text {out }}\right)$ & +0.039 & +0.073 & +0.067 & +0.006 & $-1 \cdot 10^{-5}$ \\
$\% \operatorname{dev}(H)$ & 61.302 & 59.542 & 63.042 & 1.427 & - \\
\hline
\end{tabular}

(b) Simulation example following case 2. The values of parameters are the same as in Fig. 1 for $v_{\text {in }}=1 \mathrm{~m} / \mathrm{s}$.

\begin{tabular}{|l||c|c|c|c|c|}
\hline errors & AM1 & Verlet & Heun & RK4 & $\tilde{v}_{\text {out }}$ \\
\hline$\% \operatorname{dev}(x)$ & 4.381 & 4.418 & 19.506 & 0.412 & - \\
$\% \operatorname{err}\left(v_{\text {out }}\right)$ & +2.551 & +0.839 & -4.692 & -0.105 & -0.013 \\
$\% \operatorname{dev}(H)$ & 7.885 & 9.475 & 23.387 & 0.410 & - \\
\hline
\end{tabular}

\section{IMPROVED NUMERICAL SIMULATIONS}

\section{A. Exploitation of analytical results}

In this section some solutions are proposed that allow to fix the inconsistencies pointed out in The aim is to improve the accuracy and reliability of simulations which make use of the impact model under study, in view of their implementation as real-time applications.

1) Hybrid numerical-analytical computation: This solution consists in computing the compression velocity $v_{n}$ numerically - that is as a result of a numerical simulation, using for example one of the numerical methods described in $\amalg I-\mathrm{A}$ - and employing it in (3) in order to calculate the corresponding value of compression $x\left(v_{n}\right)$ analytically. Different numerical methods may need different implementations of this solution.

As a result, the corrected numerical system strictly follows the analytical curves $x(v)$ and $H(v)$.

Since the computation is to be made at each sample for the whole duration of the contact interaction, this solution noticeably increases the computational load of the simulation.

2) Output velocity constraint: The solution above can be applied only while the contact interaction lasts, and therefore it cannot control the behavior of a numerical system upon the end of the impact. At that time, the energy content (i.e. the residual kinetic energy $T_{\tau}^{\text {sim }}=H_{\tau}^{\text {sim }}$ ) can be controlled by forcing 
the output velocity to the approximate value $\tilde{v}_{\text {out }}$.

Considering that the error introduced by (7) depends solely on the product $\mu v_{\text {in }}$ (see Fig. 3), it is advisable to apply the correction only when the product $\mu v_{\text {in }}$ corresponds to an acceptable error, or the risk is to even worsen the behavior of the numerical system. However, the use of this conditional correction always implies a trade-off: on the one hand it guarantees not to introduce errors greater than a chosen maximum, while on the other hand 1) within the excluded range of $\mu v_{\text {in }}$, the output velocity is not controlled and therefore depends only on the plain numerical method chosen, 2) within the included intervals of $\mu v_{\text {in }}$, the risk is that the correction introduces errors even greater than those provided by the non-corrected numerical system (this is true especially for non-critical parameter regions).

Once the output velocity has been forced to $\tilde{v}_{\text {out }}$, the corresponding compression should be set to 0 , this way adhering to the compression-force characteristics shown in Fig. 2 by closing their numerical counterpart at $(x=0, f=0)$, and ensuring that the final potential energy $V_{\tau}^{\text {sim }}$ is set to zero.

The computation of $\tilde{v}_{\text {out }}$ only needs to take place in correspondence to an impact event, and as soon as the impact velocity $v_{\text {in }}$ is known.

\section{B. Numerical simulations with corrections}

In order to test the described corrections, they were applied to the worst behaving simulation examples provided in $\amalg$ II-B2 Fig. 9 and 10 show a comparison of Heun-based simulations following respectively case 1 and case 2, with and without corrections.

1) Improved energy behavior: When the hybrid correction described in IV-A1 is applied, any simulation strictly adheres to the analytical curves $H(v)$ and $x(v)$ during contact, that is the respective errors, as defined in (27) and (28), are equal to zero 5

As for the energy state upon the end of the interaction, the error on $H_{\tau}$ depends either on the error introduced by $\tilde{v}_{\text {out }}$ (when the output velocity constraint described in IV-A2 is actually applied), or on the error introduced by the plain numerical simulation. As already stated in IV-A2 in the first case the maximum error on $v_{\text {out }}$ is predictable, and clearly the same goes for the error on $H_{\tau}$.

Equation (27) allows to quantitatively assess the improvements on the energy consistency of the numerical simulations.

The trend of error on $H$ resulting from simulations with and without corrections is depicted in Figures 11(a) and 11(b), which show that even the best numerical method among those considered (i.e. RK4) can be improved, especially for critical values of parameters (see पII-B2) such as $\mu v_{\text {in }} \rightarrow 0_{+}$.

\footnotetext{
${ }^{5}$ Not taking into account the inherent errors related to the representation of numbers in the digital domain.
} 


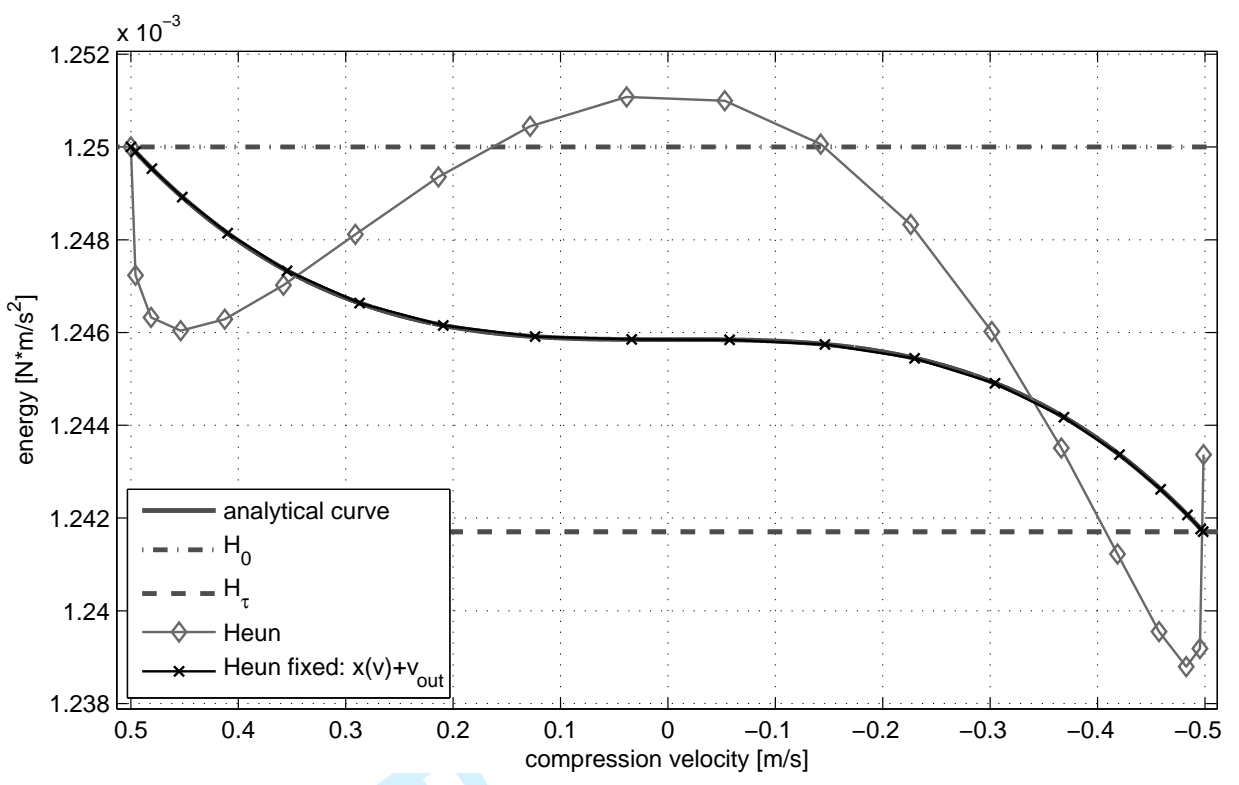

Fig. 9. Comparison of the Hamiltonians of a Heun-based simulation example following case 1, with and without corrections.

2) Sequence of impacts: In order to better appreciate the importance of the proposed corrections, a sequence of rebounds has been implemented applying a conservative force (e.g. gravity) during free motion only 6 Thanks to this setup, one can track the accumulation of energy anomalies at each contact interaction.

To this end, the residual energy $H_{\tau(i)}^{\text {sim }}$ of numerical simulations after the $i$-th impact was examined and compared to the residual energy $H_{\tau(i)}$ due to the exit velocity $v_{\text {out }}(i)$ of the $i$-th rebound, computed numerically as a zero of (6). The corresponding relative error provides a measure of the inaccuracies accumulated during the sequence of impacts. In addition, the deviation of $H^{\text {sim }}$ with respect to the analytical curve $H(v)$ was measured according to (27) along the whole sequence of impacts.

Table [II shows the errors on the residual energy $H_{\tau(100)}^{\text {sim }}$ and the maximum deviations of $H^{\text {sim }}$ occurred during a sequence of 100 impacts, for simulation examples following case 1 and case 2. Notice that, since in some simulations the energy can strongly oscillate during contact (see Fig. 7), the maximum deviations from $H(v)$ do not necessarily reflect the accumulated errors. The last columns show the errors resulting from simulations corrected as suggested in IV-A, where the error threshold for the output

\footnotetext{
${ }^{6}$ That is, the external force is temporarily suspended during the short contact at each rebound. As shown in $\amalg-\mathrm{B}$, when an external force is applied during contact, no closed-form analytical result is available, this way making the corrections provided in IV-A unsuitable.
} 


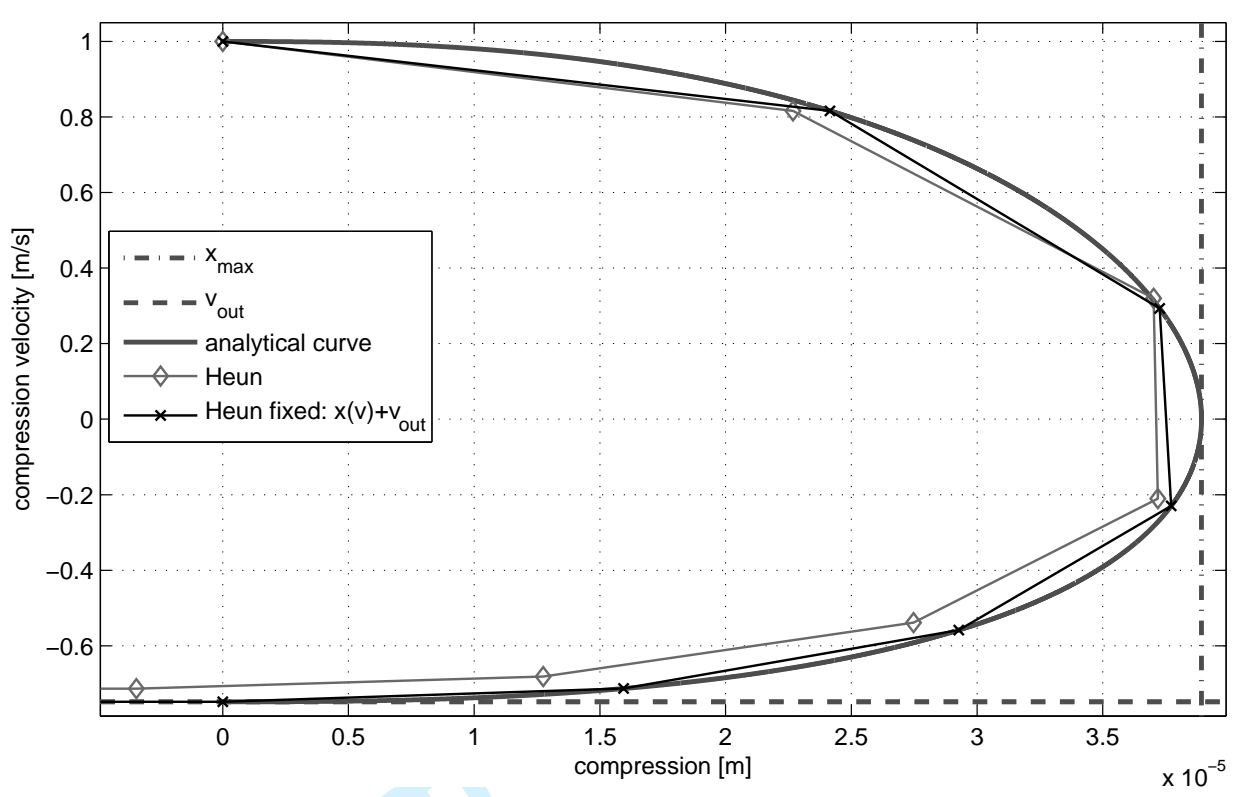

(a) Phase portraits.

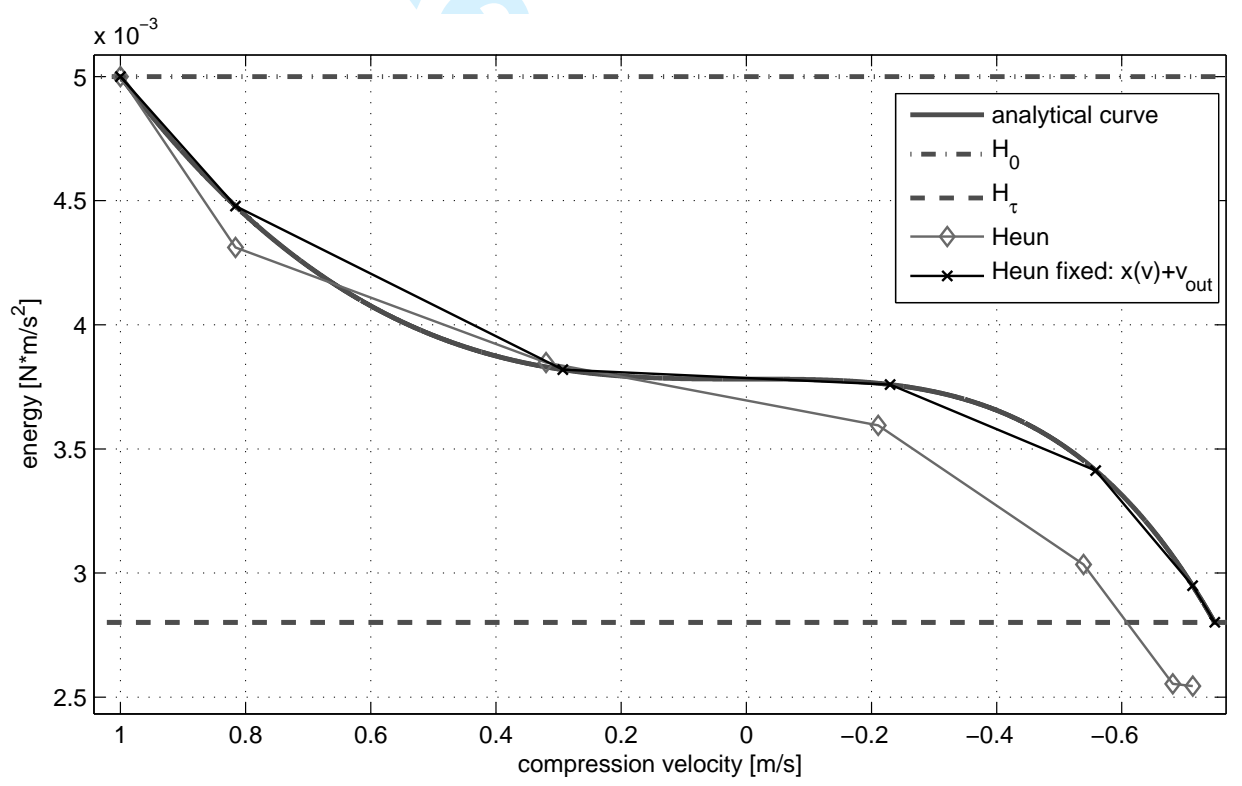

(b) Hamiltonians.

Fig. 10. Comparison of a Heun-based simulation example following case 2, with and without corrections.

velocity constraint is set to $0.1 \%$, i.e. the correction is always applied (see Fig. 3).

In order to better understand the importance of such corrections, Fig. 12 provides a partial plot of the sequence of rebounds for case 2, where only the two better performing simulations are depicted.

From Table $\amalg$ and Fig. 12 it is evident that, even in case of errors apparently negligible for a single 


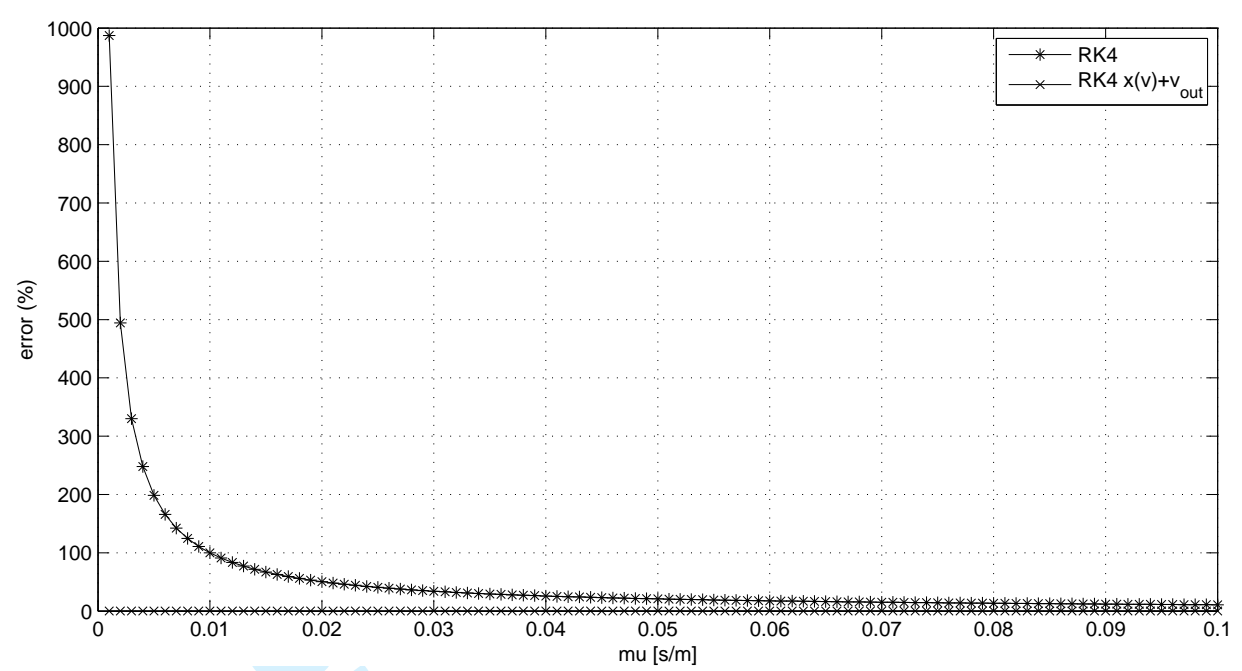

(a) Maximum deviation on $H$ as $\mu$ varies.

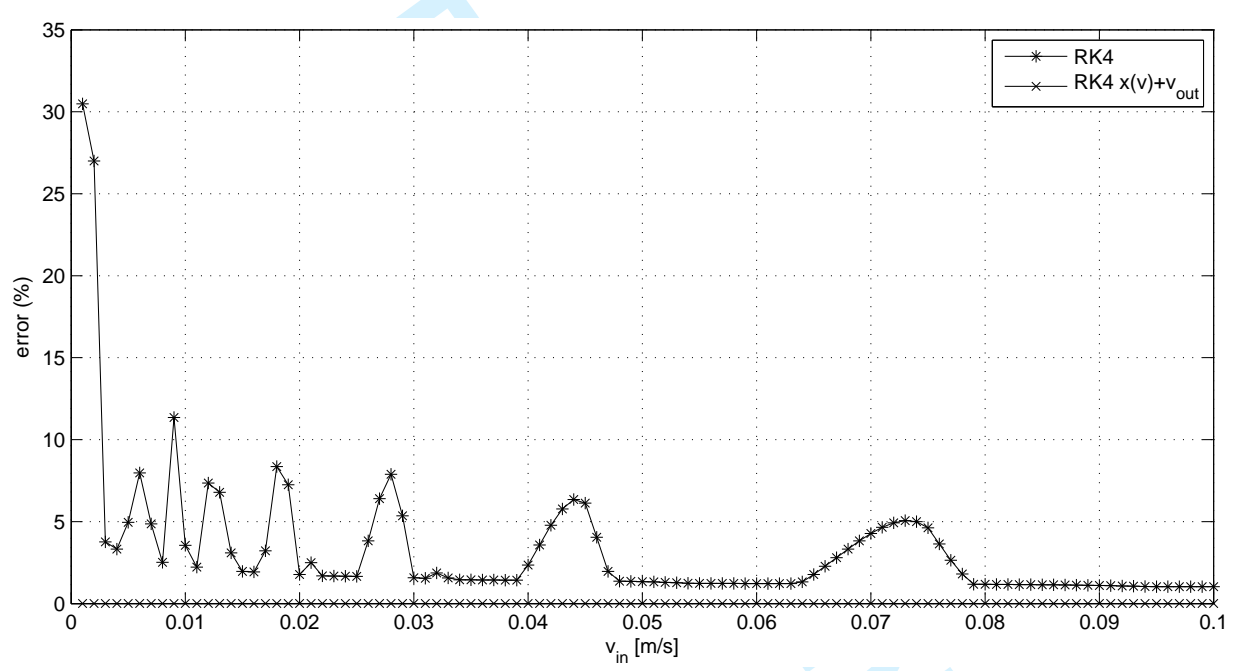

(b) Maximum deviation on $H$ as $v_{\text {in }}$ varies.

Fig. 11. Trend of error on the Hamiltonian for RK4-based simulations with and without corrections, for small values of $\mu$ and $v_{\text {in. }}$ For the values of $\mu v_{\text {in }}$ considered, the output velocity constraint is always applied (see Fig. 3). The values of parameters, where kept constant, are $k=10^{9} \mathrm{~N} / \mathrm{m}^{\alpha}, \mu=0.5 \mathrm{~s} / \mathrm{m}, \alpha=1.5, v_{\text {in }}=0.5 \mathrm{~m} / \mathrm{s}$. At each corresponding point of the two parallel plots, the product $\mu v_{\text {in }}$ is the same. It can be noted that, when not using any corrections, the weight of $\mu$ on the error is clearly greater than that of $v_{\text {in }}$.

impact (see the errors relative to RK4 in Table ПI), indeed energy inconsistencies accumulate and can become noticeable - if not disastrous - after a certain number of contact interactions.

On the other hand, the errors resulting from the corrected simulations are actually negligible, and they are only due to inaccuracies in the forced exit velocity $\tilde{v}_{\text {out }}$. The effectiveness of such corrections is 
IEEE TRANSACTIONS ON AUDIO, SPEECH, AND LANGUAGE PROCESSING, VOL. 0, NO. 0, JANUARY 0000

TABLE III

SUMMARY OF ERRORS ON THE RESIDUAL ENERGY $H_{\tau(100)}^{\text {SIM }}$ (ACCUMULATED ERROR), AND MAXIMUM DEVIATIONS OF $H^{\text {SIM }}$ CALCULATED ACCORDING TO 27] ALONG THE WHOLE SUCCESSION OF 100 REBOUNDS.

(a) Simulation examples following case 1 . The values of parameters are the same as in Fig. 7 except for input velocities decreasing at each rebound.

\begin{tabular}{|l||c|c|c|c|c|}
\hline errors on $H$ & AM1 & Verlet & Heun & RK4 & $\begin{array}{c}\text { corrected } \\
\text { sim. }\end{array}$ \\
\hline accum. \%err & 12.022 & 10.059 & 147.036 & 0.907 & $<10^{-7}$ \\
max \%dev & 72.962 & 72.107 & 63.043 & 14.466 & 0 \\
\hline
\end{tabular}

(b) Simulation examples following case 2. The values of parameters are the same as in Fig. 11 starting with input velocity $v_{\text {in }}=1 \mathrm{~m} / \mathrm{s}$.

\begin{tabular}{|l||c|c|c|c|c|}
\hline errors on $H$ & AM1 & Verlet & Heun & RK4 & $\begin{array}{c}\text { corrected } \\
\text { sim. }\end{array}$ \\
\hline accum. \%err & 24.285 & 15.780 & 946.600 & 2.153 & 0.001 \\
max \%dev & 69.156 & 43.966 & 27.418 & 6.255 & 0 \\
\hline
\end{tabular}

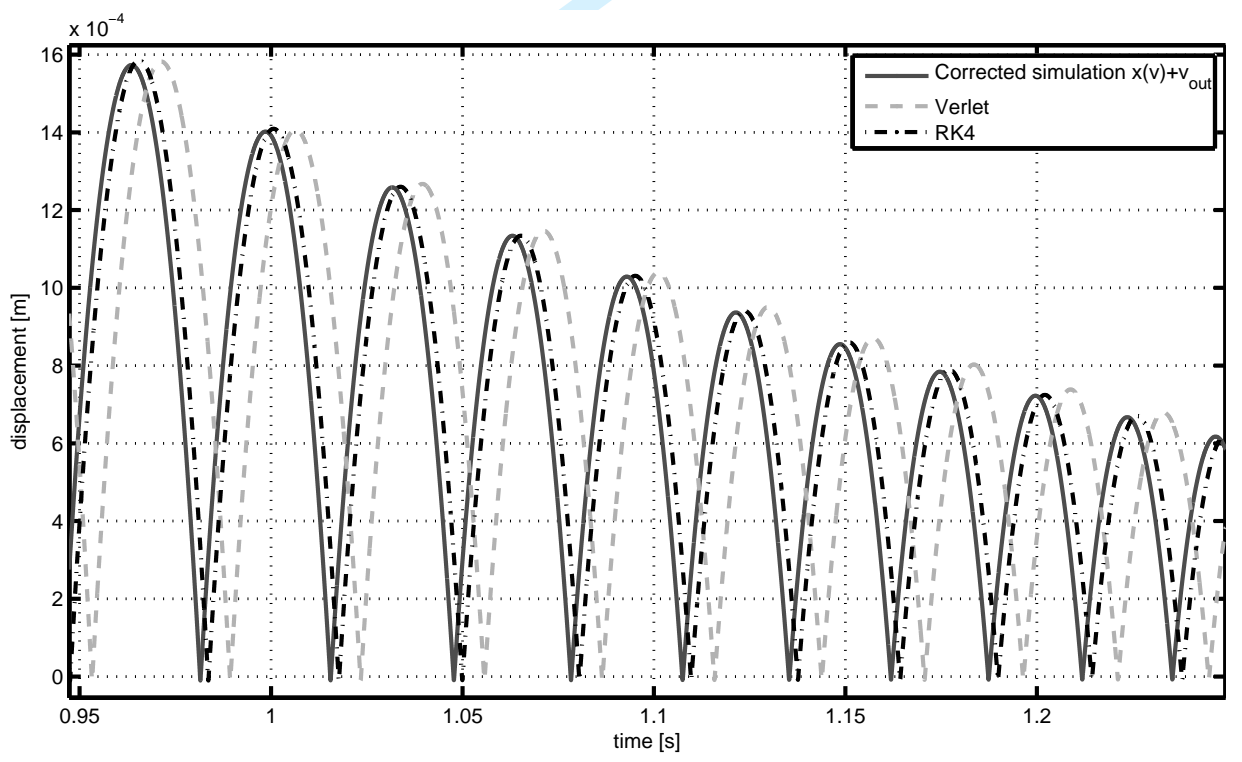

Fig. 12. Sequence of rebounds obtained from Verlet- and RK4-based simulations following case 2, compared with the trajectory of a corrected simulation.

confirmed comparing the results of a $16 \times$ oversampled RK4 simulation used as a reference: in case 1 the accumulated error on $H$ is $0.001 \%$ and the maximum deviation is $0.024 \%$, while in case 2 they are 
respectively $0.002 \%$ and $0.006 \%$.

\section{Computational cost}

In this section, the computational costs of both the numerical implementations seen in $\amalg I-\mathrm{A}$ and the corrections suggested in IV-A are taken into account. In particular, the cost has been measured as the number of operations (i.e., memory write/read accesses and arithmetical operations) needed to execute an algorithm.

Table IV(a) summarizes the cost of the algorithms implementing the numerical methods. Since the AM1-based implementation makes use of Newton's method, its cost is displayed on two sub-columns: the first one shows the constant cost per sample, while the second one (in italics) shows the cost of a single iteration of Newton's method. Notice that the number of iterations per sample is not predictable.

Table IV(b) shows the cost of the corrections considered. For comparison, the last column reports the cost of a single zero-finding iteration on (6). The number of iterations depends on $\mu$ and $v_{\text {in }}$, and is usually in the order of some tens 7 It is clear that, despite being more precise than the approximate value $\tilde{v}_{\text {out }}$, the value computed numerically as a zero of (6) implies several times the number of operations required by $\tilde{v}_{\text {out }}$.

Since the computational load of simple write/read operations is generally low (if not negligible), two totals for each column are reported: one excluding write/read operations and, in brackets, one accounting for them.

Recalling that the hybrid correction only affects the computational cost during contact, whereas the output velocity constraint is applied at mos 8 once per impact event, from Table IV] one can infer that Verlet- or Heun-based simulations with corrections are roughly three times as efficient as plain RK4based simulations during free motion, and almost twice as efficient during contact. On the other hand, while the exact computational load of an AM1-based simulation is not predictable, it can be noted that it already matches the cost of a RK4-based one after two iterations of Newton's method.

\section{Evaluation of methods}

The considered implementations can be finally evaluated in the light of the results regarding their accuracy (see III-B and IV-B2) and computational load (see IV-C).

\footnotetext{
${ }^{7}$ The number of iterations was empirically found as being usually between 15 and 40 . Moreover, as $\mu$ decreases, the number of iterations increases.

${ }^{8}$ As shown in IV-A2 the output velocity constraint is applied conditionally.
} 
TABLE IV

NUMBER OF OPERATIONS NEEDED BY THE NUMERICAL METHODS SHOWN IN II-A AND THE CORRECTIONS DESCRIBED INIV-A. THE TOTALS IN BRACKETS ACCOUNT FOR WRITE/READ OPERATIONS.

(a) Since the AM1-based implementation makes use of Newton's method, its cost is displayed on two sub-columns: the first one shows the cost per sample, while the second one (in italics) shows the cost of a single Newton's method iteration.

\begin{tabular}{|l||cc|c|c|c|}
\hline & \multicolumn{2}{|c|}{ AM1 } & Verlet & Heun & RK4 \\
\hline write & 6 & 10 & 5 & 5 & 20 \\
read & 16 & 33 & 20 & 21 & 72 \\
\hline+ I- & 3 & 8 & 7 & 8 & 22 \\
$\times$ & 8 & 11 & 5 & 5 & 18 \\
$\div$ & 0 & 1 & 3 & 3 & 8 \\
bit-shift & 0 & 0 & 2 & 3 & 6 \\
exp & 0 & 1 & 1 & 1 & 4 \\
log & 0 & 0 & 0 & 0 & 0 \\
compare & 0 & 4 & 1 & 1 & 4 \\
\hline TOTAL & $11(33)$ & $25(68)$ & $19(44)$ & $21(47)$ & $62(154)$ \\
\hline
\end{tabular}

(b) The last column (in italics) shows the cost of a single zero-finding iteration on 6 .

\begin{tabular}{|l||c|c|c|}
\hline & $\begin{array}{c}\text { hybrid } \\
\text { correct. }\end{array}$ & $\begin{array}{c}\tilde{v}_{\text {out }} \\
\text { constraint }\end{array}$ & $\begin{array}{c}v_{\text {out }} \\
\text { as zero of 66 }\end{array}$ \\
\hline write & 1 & 2 & 1 \\
read & 7 & 4 & 3 \\
\hline$+/-$ & 6 & 5 & 4 \\
$\times$ & 7 & 6 & 3 \\
$\div$ & 3 & 4 & 1 \\
bit-shift & 0 & 1 & 0 \\
exp & 0 & 1 & 0 \\
log & 1 & 0 & 1 \\
compare & 1 & 1 & 0 \\
\hline TOTAL & $18(26)$ & $18(24)$ & $9(12)$ \\
\hline
\end{tabular}

It can be stated that, among the non-corrected implementations, the best all-round performance is achieved by RK4-discretized systems, although they are quite computationally expensive. On the other hand, a corrected Verlet-discretized system is generally at least as good as a non-corrected RK4 imple- 
mentation, at a fraction of its computational load.

As for AM1-based implementations, it was shown that they behave quite poorly in the critical regions identified as case 1 and case 2. These poor results in terms of accuracy, together with a generally high (and non-predictable) computational load, set the AM1 method as a hardly recommendable choice. Apparently such conclusion can be quite surprising, especially if one considers that AM1 is the only implicit and $A$-stable method among those considered. However, the presence of nonlinearities, together with the inaccuracies introduced by the K-method and Newton's method, justify the behavior of AM1based implementations.

\section{CONCLusions}

A non-linear physical model of impact with sound synthesis applications has been reviewed, and its properties have been studied using both analytical tools and numerical simulations.

Several numerical realizations have been compared, and their shortcomings with regard to the corresponding analytical results have been pointed out. Special emphasis has been placed on energy consistency.

It has been shown that by exploiting the analytical results provided, the inconsistencies of the numerical realizations can be amended, thus restoring the correct energy state of the simulated systems, during and after contact.

Future research will consider finding a closed-form approximation of the release velocity for the system of (21), where a constant external force is applied, in this way allowing to implement suitable corrections. Even without such a closed-form solution, zero-finding numerical procedures could be profitably used to fix individual impacts. More interestingly for applications in acoustics, solutions will be investigated for extending the corrections to the case where vibrational losses are present, thus being applicable to impacts with resonating objects.

\section{APPENDIX}

\section{A. Polynomial expansion of the output velocity}

As mentioned in $\amalg-\mathrm{A} 2$. Hunt and Crossley [12] suggested that, in the limit $\mu v_{\text {in }} \rightarrow 0_{+}$, the restitution coefficient can be approximated by the linear function $\widetilde{E}\left(\mu v_{\text {in }}\right)=\left(1-2 / 3 \mu v_{\text {in }}\right)$. Then, recalling (5), the corresponding output velocity is:

$$
\tilde{v}_{\text {out }}\left(\mu, v_{\text {in }}\right)=\left(-v_{\text {in }}+\frac{2}{3} \mu v_{\text {in }}^{2}\right) .
$$

This result can be easily verified through a Taylor expansion of the two sides in (6), using the approximation $\log |1+\epsilon| \approx \epsilon-\frac{\epsilon^{2}}{2}+\frac{\epsilon^{3}}{3}$, for $\epsilon \rightarrow 0_{+}$. 
The same approach can be used in order to find a polynomial expansion of order $n$ :

$$
\tilde{v}_{\text {out }}=\sum_{j=1}^{n} a_{j} \cdot v_{\text {in }}^{j}
$$

in the limit $\mu v_{\text {in }} \rightarrow 0_{+}$. Equation (30) is then substituted into the left-hand side of the Taylor expansion of (6). For the case $n=4$ one obtains:

$$
\begin{aligned}
& {\left[\frac{\mu^{2}}{2} v_{\text {out }}^{2}-\frac{\mu^{3}}{3} v_{\text {out }}^{3}+\frac{\mu^{4}}{4} v_{\text {out }}^{4}-\frac{\mu^{5}}{5} v_{\text {out }}^{5}\right]_{v_{\text {out }}=\sum_{1}^{4} a_{j} v_{\text {in }}^{j}}} \\
& =\frac{1}{2} \mu^{2} v_{\text {in }}^{2}-\frac{1}{3} \mu^{3} v_{\text {in }}^{3}+\frac{1}{4} \mu^{4} v_{\text {in }}^{4}-\frac{1}{5} \mu^{5} v_{\text {in }}^{5} .
\end{aligned}
$$

The coefficients $a_{j}$ are then determined by equating the two sides of 31 term by term, leading to the system:

$$
\left\{\begin{array}{l}
\frac{1}{2} \mu^{2} a_{1}^{2}=\frac{1}{2} \mu^{2} \\
\frac{1}{3} \mu^{3} a_{1}^{3}-\mu^{2} a_{1} a_{2}=\frac{1}{3} \mu^{2} \\
\frac{1}{4} \mu^{4} a_{1}^{4}-\mu^{3} a_{1}^{2} a_{2}+\mu^{2} a_{1} a_{3}+\frac{1}{2} \mu^{2} a_{2}^{2}=\frac{1}{4} \mu^{2} \\
\frac{1}{5} \mu^{5} a_{1}^{5}-\mu^{4} a_{1}^{3} a_{2}+\mu^{3} a_{1} a_{2}^{2}+ \\
\quad+\mu^{3} a_{1}^{2} a_{3}-\mu^{2} a_{1} a_{4}-\mu^{2} a_{2} a_{3}=\frac{1}{5} \mu^{2}
\end{array}\right.
$$

which yields:

$$
a_{1}=-1, \quad a_{2}=\frac{2}{3} \mu, \quad a_{3}=-\frac{4}{9} \mu^{2}, \quad a_{4}=\frac{44}{135} \mu^{3} .
$$

It follows that, in (30), $v_{\text {out }}$ is a function of $\left(\mu, v_{\text {in }}\right)$ only. Equations (30) and (33) result in a slightly better approximation than (29), but still provide a local approximation.

\section{B. A non-local approximation for the output velocity}

From (6) $v_{\text {out }}$ can be written as:

$$
\begin{aligned}
v_{\text {out }} & =\frac{1}{\mu}\left[\left(1+\mu v_{\text {in }}\right) e^{-\mu \Delta v}-1\right]= \\
& =v_{\text {lim }}\left[1-\left(1+\mu v_{\text {in }}\right) e^{-\mu \Delta v}\right]
\end{aligned}
$$

where $\Delta v=v_{\text {in }}-v_{\text {out }}$. Equation (34) emphasizes that the convergence $v_{\text {out }} \rightarrow v_{\text {lim }}$ for $v_{\text {in }} \rightarrow+\infty$ is governed by the fast-decreasing term $e^{-\mu \Delta v}$.

For small $v_{\text {in }}$ 's, the zeroth order approximation of the exponential term is $e^{-2 \mu v_{\text {in }}}$, however it is easy to verify that the equation:

$$
\tilde{v}_{\text {out }}=v_{\lim }\left[1-\left(1+\mu v_{\text {in }}\right) e^{-2 \mu v_{\text {in }}}\right]
$$


does not provide an accurate approximation of $v_{\text {out }}$ for small $v_{\text {in }}$ 's. More precisely, its Taylor expansion around $v_{\text {in }}=0$ only matches the first Taylor coefficient of the expansion (30):

$$
\begin{aligned}
\tilde{v}_{\text {out }} \underset{v_{\text {in }} \rightarrow 0_{+}}{\approx} \sum_{j=1}^{n} \tilde{a}_{j} \cdot v_{\text {in }}^{j}, \\
\tilde{a}_{1}=-1 \quad \text { and } \quad \tilde{a}_{j} \neq a_{j} \quad(j>1) .
\end{aligned}
$$

This qualitative discussion justifies to some extent the non-local approximation for $v_{\text {out }}$ given in (7). Indeed (7) combines the two views $-\mu v_{\text {in }} \rightarrow 0_{+}$and $\mu v_{\text {in }} \rightarrow+\infty-$ on $v_{\text {out }}$ : the exponential term ensures the convergence $v_{\text {out }} \rightarrow v_{\text {lim }}$ for high values of $\mu v_{\text {in }}$, while the polynomial coefficients $b_{j}$ are determined by imposing that the Taylor expansion of (7) around $v_{\text {in }}=0$ matches that of (30). Recalling that:

$$
e^{-2 \mu v_{\text {in }}} \underset{v_{\text {in }} \rightarrow 0_{+}}{\approx} \sum_{j=1}^{n} c_{j} \cdot v_{\text {in }}^{j}, \quad c_{j}=\frac{(-1)^{j}}{j !}(2 \mu)^{j},
$$

then one can verify that the $l$-th order coefficient $a_{l}$ of the Taylor expansion of (7) is given as $a_{l}=$ $\frac{1}{\mu} \sum_{j=1}^{l} b_{j} \cdot c_{(l-j)}$. Then the coefficients $b_{j}$ can be determined recursively as:

$$
b_{0}=1, \quad b_{j}=\mu a_{j}-\sum_{l=0}^{j-1} b_{l} \cdot c_{(j-l)} \quad(1 \leq j \leq n) .
$$

Applying this recursive equation for the case $n=4$ yields (8).

\section{REFERENCES}

[1] D. W. Marhefka and D. E. Orin, "A compliant contact model with nonlinear damping for simulation of robotic systems," IEEE Trans. on Systems, Man, and Cybernetics - Part A: Systems and Humans, vol. 29, no. 6, pp. 566-572, November 1999.

[2] M. Mahvash, V. Hayward, and J. Lloyd, "Haptic rendering of tool contact," in Proc. Eurohaptics, 2002, pp. 110-115.

[3] M. Müller, J. Stam, D. James, and N. Thürey, "Real time physics: class notes," in SIGGRAPH '08: ACM SIGGRAPH 2008 classes. New York, NY, USA: ACM, 2008, pp. 1-90.

[4] A. Chaigne and J. Kergomard, Acoustique des Instruments de Musique. Belin, 2008.

[5] F. Avanzini and D. Rocchesso, "Modeling collision sounds: non-linear contact force," in Proc. Int. Conf. on Digital Audio Effects (DAFx-01), Limerick, December 2001, pp. 61-66.

[6] M. Rath and D. Rocchesso, "Continuous sonic feedback from a rolling ball," IEEE MultiMedia, vol. 12, no. 2, pp. 60-69, 2005.

[7] D. Rocchesso and F. Fontana, Eds., The Sounding Object. Mondo Estremo, 2003. [Online]. Available: http://www.soundobject.org/

[8] P. Flores, J. P. Claro, and H. M. Lankarani, Kinematics and Dynamics of Multibody Systems with Imperfect Joints: Models and Case Studies. Springer, 2008.

[9] G. Kuwabara and K. Kono, "Restitution coefficient in a collision between two spheres," Jap. J. of Appl. Phys., vol. 26, no. 8, pp. 1230-1233, 1987. 
[10] A. Stulov, "Dynamic behavior and mechanical features of wool felt," Acta Mechanica, vol. 169, no. 1, pp. 13-21, 2004.

[11] L. Vu-Quoc and X. Zhang, "An elastoplastic contact force-displacement model in the normal direction: displacement-driven version," Proc.: Mathematical, Physical and Engineering Sciences, pp. 4013-4044, 1999.

[12] K. H. Hunt and F. R. E. Crossley, "Coefficient of restitution interpreted as damping in vibroimpact," ASME J. Applied Mech., pp. 440-445, June 1975.

[13] N. Diolaiti, C. Melchiorri, and S. Stramigioli, "Contact impedance estimation for robotic systems," IEEE Trans. on Robotics, vol. 21, no. 5, pp. 925-935, 2005.

[14] H. M. Lankarani and P. E. Nikravesh, "A contact force model with hysteresis damping for impact analysis of multibody systems," J. of Mechanical Design, vol. 112, no. 3, pp. 369-376, 1990.

[15] P. Flores, J. Ambrósio, J. Claro, and H. Lankarani, "Influence of the contact-impact force model on the dynamic response of multi-body systems," Proc. of the Institution of Mechanical Engineers-K, vol. 220, no. 1, pp. 21-34, 2006.

[16] L. Pust and F. Peterka, "Impact oscillator with Hertz's model of contact," Meccanica, vol. 38, no. 1, pp. 99-116, 2003.

[17] S. Papetti, F. Fontana, M. Civolani, A. Berrezag, and V. Hayward, "Audio-tactile display of ground properties using interactive shoes," in Proc. 5th Int. Workshop on Haptic and Audio Interaction Design (HAID '10), Copenhagen, Denmark, 2010, accepted.

[18] S. Bilbao, "Robust physical modeling sound synthesis for nonlinear systems," Signal Processing Magazine, IEEE, vol. 24, no. 2, pp. 32-41, March 2007.

[19] I. Millington, Game physics engine development. Morgan Kaufmann, 2007.

[20] J. E. Colgate and J. M. Brown, "Factors Affecting the Z-Width of a Haptic Display," in Proc. IEEE Int. Conf. on Robotics \& Automation, San Diego, May 1994, pp. 3205-3210.

[21] K. Kuchenbecker, J. Fiene, and G. Niemeyer, "Improving contact realism through event-based haptic feedback," IEEE Trans. on Visualization and Computer Graphics, vol. 12, no. 2, pp. 219-230, March-April 2006.

[22] S. Bilbao, Numerical Sound Synthesis. New York: Wiley, 2009.

[23] F. Avanzini and D. Rocchesso, "Physical modeling of impacts: theory and experiments on contact time and spectral centroid," in Proc. Int. Conf. on Sound and Music Computing (SMC'04), 2004, pp. 287-293.

[24] B. L. Giordano, D. Rocchesso, and S. McAdams, "Integration of acoustical information in the perception of impacted sound sources: The role of information accuracy and exploitability," Journal of Experimental Psychology: Human Perception and Performance, vol. 36, no. 2, pp. 462-476, 2010.

[25] A. Quarteroni, R. Sacco, and F. Saleri, Numerical Mathematics, 2nd ed. Springer, 2007.

[26] G. Borin, G. De Poli, and D. Rocchesso, "Elimination of Delay-Free Loops in Discrete-Time Models of Nonlinear Acoustic Systems," IEEE Trans. on Speech and Audio Processing, vol. 8, no. 5, pp. 597-605, September 2000. 


\begin{abstract}
A physically-based impact model - already known and exploited in the field of sound synthesis - is studied using both analytical tools and numerical simulations. It is shown that the Hamiltonian of a physical system composed of a mass impacting on a wall can be expressed analytically as a function of the mass velocity during contact. Moreover, an efficient and accurate approximation for the mass outbound velocity is presented, which allows to estimate the Hamiltonian at the end of the contact. Analytical results are then compared to numerical simulations obtained by discretizing the system with several numerical methods. It is shown that, for some regions of the parameter space, the trajectories of the discretized systems may significantly drift from the analytically-derived curves. Two approaches, based on enforcing numerical energy consistency, are then proposed to improve the accuracy of numerical simulations.
\end{abstract}

Index Terms-Real time systems, simulation.

\section{INTRODUCTION}

$\mathbf{P}$ HYSICAL models of impacts between objects are ubiquitous in many areas of science and engineering, including robotics [1], haptics [2], computer graphics [3], acoustics [4] and sound synthesis [5]. The phenomenologically plausible and energy-consistent behavior of contacting bodies is especially crucial in simulations of interactions based on sustained or repeated impacts, such as in rolling [6], scraping, or bouncing [7].

The higher is the upper limit of the perceptual bandwidth (and the rendering rate), the more critical is the accuracy that real-time numerical simulations can afford, thus making the problem of impact modeling increasingly complex when moving from graphic, to haptic, to auditory displays. Whereas for most graphic displays it is sufficient to describe an impact in terms of the ratio between outbound and inbound velocities, in haptic display and in sound synthesis the perceived characteristics of the impact depend on how bodies interact during contact. Therefore, more sophisticated impact models and carefully-designed discretizations are necessary in audio and haptic contexts.

Manuscript received January 0, 0000; revised January 0, 0000.

The research leading to these results has received funding from the EU's Seventh Framework Programme under FET-Open grant agreement $n^{\circ} 222107$ NIW - Natural Interactive Walking, http://www.niwproject.eu

S. Papetti is with the Dip. Informatica, University of Verona. e-mail: stefano.papetti@univr.it

F. Avanzini is with the Dip. Ingegneria dell'Informazione, University of Padova. e-mail: avanzini@dei.unipd.it

D. Rocchesso is with the Dip. Arti e Disegno Industriale, IUAV University of Venice. e-mail: roc@iuav.it

\section{A. Impact Models}

The classic starting point is the Hertz model of collision between two spheres, which can be extended to include internal viscosity [8]. The impact force in such models is the sum of a nonlinear elastic term - in the form of a power law of compression - and a dissipative component proportional to the compression velocity - via a second power law of compression. The exponents of the two power laws, as derived for two colliding spheres, are $3 / 2$ and $1 / 2$, respectively [9].

In the context of musical acoustics, Stulov proposed a piano hammer model that includes relaxation properties of felt [10]. Such model has exponents $\alpha$ and $\alpha-1$ for the power laws, and the actual value of $\alpha$ can be used to match experimental data. Other models exist that take plastic deformations into account, thus introducing abrupt direction changes in the forcecompression curves at the transition between loading and unloading [11].

Particularly popular is the model by Hunt and Crossley [12], [1], [13], [14], [15], that generalizes the extended Hertz model by considering a variable exponent that accounts for different contact shapes. In this model, the power laws in the elastic and dissipative term are considered to be equal, thus allowing easier closed-form calculations [16]. Despite not being fully justified in physical terms, the Hunt-Crossley model has been quite successful in some areas of engineering because it allows to derive the phase trajectories in closed form, and because it is sufficiently complex to represent a wide variety of contact phenomena. In our work, we adopted this model and extended the range of the available analytical results.

\section{B. Applications in acoustic modeling}

Contact models can serve as a basis for developing models of acoustic phenomena. In the context of physically-based sound synthesis, the Hunt-Crossley model has been used to develop an impact sound model [5], where a generic resonating object is used in place of the classic rigid wall.

Other models of more complex acoustic phenomena have been developed based on the very same impact model studied here. As an example, a bouncing sound model [7] has been obtained by superimposing a constant force, which simulates gravity, on a plain impact sound model. Also, a rolling sound model [6] has been implemented by driving an impact sound model by means of a physically-inspired control layer. More precisely, the continuous interaction of a ball rolling on a surface has been modeled as a dense temporal sequence 
of micro-impacts driven by the geometry of the contacting surfaces, and modulated by the ball's asymmetry.

Accurate and consistent impact modeling is crucial in some audio-haptic rendering applications, such as interactive floors or shoes with vibratory and sonic augmentation [17].

In the context of musical sound synthesis, the piano and other percussive musical instruments have also been modeled by using dissipative impact models [10].

\section{Issues with discrete time}

A wide range of numerical methods can be employed to discretize the interaction of impacting bodies. Given a reference continuous-time system, such as the Hunt-Crossley model, the goal is to obtain numerical quantities that follow the continuous-time trajectories as closely as possible, at an affordable computational cost. Thus, efficiency and accuracy are central issues.

Another important goal is energy consistency, especially in the case of repeated or sustained contacts. A numerical method, albeit being accurate, can introduce spurious oscillations or instabilities if it fails in terms of energy conservation. This justifies the development of energy-based methods, i.e. numerical schemes based on the definition of a numerical energy that is proved to be conserved in lossless conditions [18]. These provably-stable discrete-time models can be derived for some continuous-time models, including some nonlinear oscillators, but are not available for more general nonlinear contact models.

As for applications which make use of impact/contact models, energy inconsistencies are a recurring issue. In computer graphics, where the constraint of low frame rates makes numerical systems prone to instabilities [3], a typical example is provided by a steady object in resting contact with a rigid floor: when the system does not retain passivity, the object can move upward and bounce [19]. Similar issues are encountered in simulations of haptic contact, where stiffness values are usually limited by requirements on system passivity [20], [21], whereas higher values can cause the system to become unstable, for example oscillating, or reacting actively to the input. In numerical sound synthesis by physical models [22], artifacts and inconsistencies can become audible especially in situations of sustained or repeated contact interactions, as in rolling, sliding or bouncing.

Instead of aiming at provably-stable numerical methods, this study looks at the accuracy of some methods commonly found in physics-based engines [3], and measure their performance in following the theoretical phase trajectories and in reproducing the energy exchanges that occur in the continuous-time impact model.

\section{Outline}

In this paper, the Hunt-Crossley impact model is first characterized by proving some novel analytical results. Such results are then used as a reference to compare the accuracy of several numerical simulations of the model, obtained by discretizing the continuous-time equations with a number of widely used numerical methods. It is shown that for some regions of the parameter space, the trajectories of the discretized systems may significantly drift from the analyticallyderived curves. Finally, by exploiting the provided analytical results, two approaches are proposed which allow to improve the accuracy of the numerical simulations, thus restoring their energy consistency.

More in detail, the main contribution of this work can be summarized as follows:

1) Novel approximate closed-form expression for the outbound velocity, and quantification of its relative error. Expression of the total energy (Hamiltonian) as a function of compression velocity (Section $\llbracket-\mathrm{A}$ ).

2) Analysis of the distortion caused by a constant external force on the analytically derived phase portraits. This is of some importance for applying the results to real-world simulations (Section ஹ-B).

3) Comparison of the analytically-derived phase portrait and Hamiltonian with those obtained by applying four relevant numerical methods to the Hunt-Crossley impact model. Two critical cases are examined: low dissipation and hard impact (Section II-B).

4) Proposal of two correction methods for numerical models, one based on the analytic dependence of compression on velocity, and one based on a constraint on the outbound velocity (Section IV).

The remainder of the paper is organized as follows. Section $\Pi$ contains the analytical study, Section III-A describes different numerical methods which are used for discretizing the continuous-time system, while in Section $\Pi$ I-B the corresponding numerical simulations are compared. Sections IV-A and IV-B show how the provided analytical results can be used to improve the behavior of the numerical simulations. Finally, in Sections IV-C and IV-D the computational cost and accuracy of simulations with and without corrections are compared and evaluated.

\section{IMPACT MODEL}

The Hunt-Crossley impact model [12] is described by the following non-linear equation describing the impact force:

$$
f(x, v)= \begin{cases}k x^{\alpha}+\lambda x^{\alpha} v=k x^{\alpha} \cdot(1+\mu v), & x>0 \\ 0 & , \quad x \leq 0\end{cases}
$$

where $x$ is the compression, $v=\dot{x}$ is the compression velocity, $\alpha>1$ is the exponent of a power law and represents the local shape of contact surfaces, $k$ is the stiffness coefficient, and $0 \leq \lambda \leq k$ is the damping coefficient. The mathematically convenient term $\mu(=\lambda / k)$ allows to simplify some closedform calculations. The impact force model thus represents a non-linear spring of constant $k$ in parallel with a nonlinear damper of constant $\lambda$. The term $k x^{\alpha}$ corresponds to the elastic component, while $\lambda x^{\alpha} v$ represents the dissipation due to internal friction.

Marhefka and Orin [1] made use of the Hunt-Crossley model in order to represent the impact between a lumped point-mass and a rigid wall (representing a comparatively massive surface which does not move during collision), therefore considering the system described by the equation:

$$
m a(t)=-f(x(t), v(t))
$$




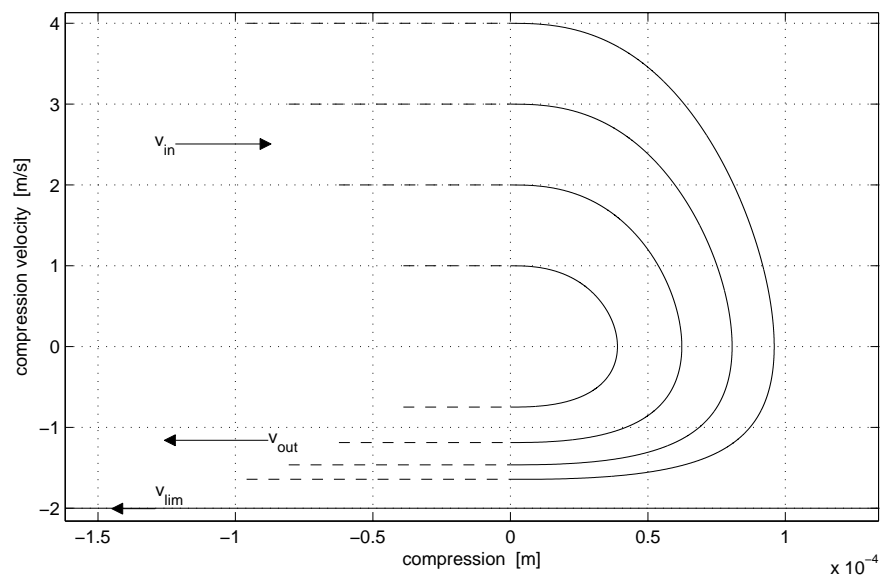

Fig. 1. Phase portraits for varying input velocities: $v_{\text {in }}=1 \ldots 4 \mathrm{~m} / \mathrm{s}$. Other values of parameters are: $m=10^{-2} \mathrm{~kg}, k=10^{9} \mathrm{~N} / \mathrm{m}^{\alpha}, \mu=0.5 \mathrm{~s} / \mathrm{m}$, $\alpha=1.5$. Solid lines represent the mass trajectory during contact; dashed lines represent free motion.

where $m$ is the mass, and $a$ is its acceleration. In this basic case, during contact the compression and the compression velocity are respectively equivalent to the displacement and the velocity of the point-mass.

\section{A. Properties and analytical results}

Thanks to the simple form of (2), the model can be treated analytically and some of its properties can be inferred. Hereafter the initial conditions $x(0)=0$ and $\dot{x}(0)=v_{\text {in }}$ are considered, that is to say that the point-mass hits the rigid wall with velocity $v_{\text {in }}$ at time $t=0$.

1) Compression: It is shown in [1] that from (2) it follows: $x(v)=\left[\frac{m(\alpha+1)}{k \mu^{2}} \cdot\left(-\mu\left(v-v_{\text {in }}\right)+\log \left|\frac{1+\mu v}{1+\mu v_{\text {in }}}\right|\right)\right]^{\frac{1}{\alpha+1}}$

which can be exploited for plotting the phase portraits on the $(x, v)$ plane shown in Fig. 1 . From Fig. 1 it can be inferred that, due to the viscous dissipation occurring during contact, the relation $v(t+d t)<v(t)$ holds, and in particular the output velocity $v_{\text {out }}$ is always smaller in magnitude than the corresponding $v_{\text {in }}$. Moreover, for increasing $v_{\text {in }}$ 's, $v_{\text {out }}$ converges to the limit value $v_{\text {lim }} \triangleq-1 / \mu$. The line $v=v_{\text {lim }}$ represents the trajectory where the elastic and dissipative terms cancel, and separates two regions of the phase space, each of which is never entered by trajectories started in the other one.

Equation (3) allows to infer the maximum compression experienced during contact, which occurs when the compression velocity equals zero:

$$
x_{\text {max }}=x(0)=\left[\frac{m(\alpha+1)}{k \mu^{2}} \cdot\left(\mu v_{\text {in }}+\log \left|\frac{1}{1+\mu v_{\text {in }}}\right|\right)\right]^{\frac{1}{\alpha+1}} .
$$

As remarked in [1], (1) together with Fig. 11 show that the force $f$ becomes sticky (inward) when $v<v_{\text {lim. However there }}$ is no physical inconsistency in this "stickiness" property, and indeed this never occurs for trajectories with initial conditions $x(0)=0$ and $\dot{x}(0)=v_{\text {in }}>0$.

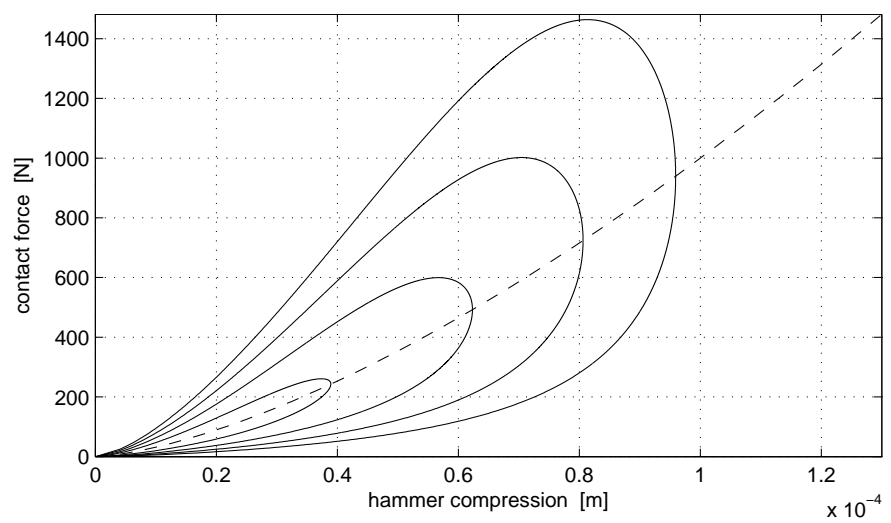

Fig. 2. Compression-force characteristics for varying input velocities: $v_{\text {in }}=1 \ldots 4 \mathrm{~m} / \mathrm{s}$. Solid lines represent the case when dissipation is taken into account (the values of parameters are the same as in Fig. 1. The dashed line represents the curve $k x^{\alpha}$, where no dissipation is considered $(\lambda=0)$.

Finally, by substituting (3) in (2) the compression-force characteristics during collision can be plotted, which are shown in Fig. 2. It can be noted that the dissipative term $\lambda x^{\alpha} v$ introduces hysteresis around the curve $k x^{\alpha}$.

2) Output velocity: The restitution coefficient $E$ is defined as:

$$
E \triangleq\left|\frac{v_{\text {out }}}{v_{\text {in }}}\right| \text {. }
$$

Note that $v_{\text {in }}$ and $v_{\text {out }}$ correspond to the roots of the right-hand side of (3), that is the points where $x=0$. As a result, $v_{\text {out }}$ can be defined implicitly from (3) as a function of $\left(\mu, v_{\text {in }}\right)$ only:

$$
\mu v_{\text {out }}-\log \left|1+\mu v_{\text {out }}\right|=\mu v_{\text {in }}-\log \left|1+\mu v_{\text {in }}\right| .
$$

This implies that $\mu v_{\text {out }}$ is a function of $\mu v_{\text {in }}$ only, and therefore $E$ is also a function of $\mu v_{\text {in }}$ only.

Analytical derivations of the dependence $E\left(\mu v_{\text {in }}\right)$ have been classically performed in the limit of small initial velocities and/or small dissipation [12]1 However, we suggest that a non-local approximation $\tilde{v}_{\text {out }}$ can be empirically determined as an ansatz which fits the curve $E\left(\mu v_{\text {in }}\right)$ in the two limit regions $\mu v_{\text {in }} \rightarrow 0_{+}$and $\mu v_{\text {in }} \rightarrow+\infty$, thus obtaining 2 :

$$
\tilde{v}_{\text {out }}\left(\mu, v_{\text {in }}\right)=v_{\text {lim }}\left[1-\left(\sum_{j=0}^{n} b_{j} \cdot v_{\text {in }}^{j}\right) e^{-2 \mu v_{\text {in }}}\right]
$$

where, in the case $n=4$, the coefficients $b_{j}$ are:

$b_{0}=1, \quad b_{1}=\mu, \quad b_{2}=\frac{2}{3} \mu^{2}, \quad b_{3}=\frac{2}{9} \mu^{3}, \quad b_{4}=\frac{14}{135} \mu^{4}$.

From now on, unless specified otherwise, the notation $\tilde{v}_{\text {out }}$ will refer to the fourth-order approximation provided by (7) and the coefficients (8). Conventional iterative zero-finding methods applied to (6) can always be used to compute a more precise release velocity at a higher computational cost (see IV-C).

Fig. 3 shows the error introduced by the approximate value $\tilde{v}_{\text {out }}$, when compared to the corresponding value computed numerically as a zero of (6).

\footnotetext{
${ }^{1}$ Appendix $\mathrm{A}$ provides an example.

${ }^{2}$ See Appendix B for details.
} 


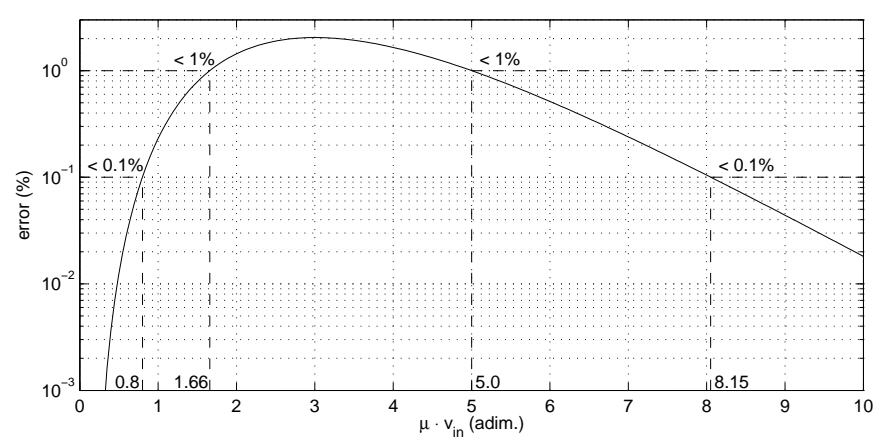

Fig. 3. Log-scaled percentage error of the output velocity approximated by $\tilde{v}_{\text {out }}$, with respect to the value computed numerically as a zero of 6. Ranges of $\mu v_{\text {in }}$ are shown, for which the maximum error is respectively less than $1 \%$ and $0.1 \%$.

3) Contact time: It is shown in [23] that the contact time can be expressed as:

$$
\begin{aligned}
& \tau=\left(\frac{m}{k}\right)^{\frac{1}{\alpha+1}} \cdot\left(\frac{\mu^{2}}{\alpha+1}\right)^{\frac{\alpha}{\alpha+1}} \cdot \\
& \cdot \int_{v_{\text {out }}}^{v_{\text {in }}} \frac{1}{(1+\mu v)\left[-\mu\left(v-v_{\text {in }}\right)+\log \left|\frac{1+\mu v}{1+\mu v_{\text {in }}}\right|\right]^{\frac{\alpha}{\alpha+1}}} .
\end{aligned}
$$

Equation (9) states that the contact time $\tau$ depends only on $\mu$, the exponent $\alpha$ and the ratio $m / k$, plus obviously the impact velocity $v_{\text {in }}$. Since neither $m$ nor $k$ affect the value of the integral (recall that $v_{\text {out }}$ depends only on $\mu$ and $v_{\text {in }}$ ), it follows that, given a fixed $v_{\text {in }}$, the proportionality $\tau \sim(m / k)^{1 /(\alpha+1)}$ holds.

From an auditory point of view the value of the contact time is strongly correlated to the perceived "hardness" of the impact [23], [24]. Namely, as the contact time decreases, the perceived hardness increases. Recalling the power-law dependence above and (1) it follows that, for a fixed mass $m$, "hard" and "soft" impacts correspond respectively to high and low force values.

4) Energy properties and behavior: The energy variation in a mechanical system can be calculated as the work made by the non-conservative forces $f_{\mathrm{nc}}$ acting on the system along a certain path $x_{1} \rightarrow x_{2}$ :

$$
\Delta H=\int_{x_{1}}^{x_{2}} f_{\mathrm{nc}}(x) d x=\int_{t_{1}}^{t_{2}} f_{\mathrm{nc}}(t) v(t) d t=-\Delta \Lambda
$$

where $H$ is the total energy content, known as the Hamiltonian, $\Lambda$ is the energy dissipation, and the second integral is obtained by considering that $t_{1}$ and $t_{2}$ correspond respectively to the instants when the displacements $x_{1}$ and $x_{2}$ are reached. The Hamiltonian $H$ is the sum of potential and kinetic energies, hereafter named $V$ and $T$, respectively:

$$
H(t)=V(t)+T(t) .
$$

With regard to the system represented by (2), $T$ is related to the dynamics of the point-mass, which is described by the lefthand side of (2), while $V$ is related to the elastic component of the impact force of (1).
In agreement with the last integral in 10, multiplying both sides of (2) by $v(t)=d x / d t$ and time-integrating them, gives:

$$
\begin{aligned}
& \underbrace{\int_{0}^{t} m a(s) v(s) d s}_{T(t)-T_{0}}= \\
& =-\underbrace{\int_{0}^{t} k x(s)^{\alpha} v(s) d s}_{V(t)}-\underbrace{\int_{0}^{t} \lambda x(s)^{\alpha} v(s)^{2} d s}_{\Lambda(t)>0}
\end{aligned}
$$

where the force expression of (1) has been considered in the case $x>0$ only. The first two integrals in (12) can be solved explicitly, obtaining:

$$
V(t)=\frac{k x(t)^{\alpha+1}}{\alpha+1}, \quad T(t)=\frac{m v(t)^{2}}{2} .
$$

Considering a system where the point-mass travels with velocity $v_{\text {in }}$ before an impact occurs, then the initial Hamiltonian corresponds to the initial kinetic energy:

$$
H_{0}=T_{0}=\frac{m v_{\mathrm{in}}^{2}}{2}
$$

From (10) it follows that at each time instant $t$ :

$$
H(t)=H_{0}-\Lambda(t)
$$

and, since $\Lambda(t)>0$, the following inequalities hold:

$$
0 \leq H(t+d t) \leq H(t)
$$

Indicating $\tau$ as the instant when an impact ends, the final Hamiltonian of the system, that is the energy content right after contact, can be written as:

$$
H_{\tau}=T_{\tau}=\frac{m v_{\mathrm{out}}^{2}}{2} .
$$

Also, the total amount of energy dissipation occurred during contact is:

$$
\Delta H_{\tau}=H_{\tau}-H_{0}=-\Lambda_{\tau}
$$

which corresponds to the area enclosed by the hysteresis loops shown in Fig. 2 .

As for the rightmost integral in (12), which is non-solvable, an equivalent expression can be obtained by substituting the complementary results for the remaining integrals:

$$
\Lambda(t)=\int_{0}^{t} \lambda x(s)^{\alpha} v(s)^{2} d s=\frac{m\left(v_{\mathrm{in}}^{2}-v(t)^{2}\right)}{2}-\frac{k x(t)^{\alpha+1}}{\alpha+1}
$$

Finally, by substituting (3) in (19) and recalling (15), the following expression in $v$ only is found:

$H(v)=H_{0}-\Lambda(v)=\frac{m}{2} v^{2}-\frac{m}{\mu}\left(v-v_{\text {in }}\right)+\frac{m}{\mu^{2}} \log \left|\frac{1+\mu v}{1+\mu v_{\text {in }}}\right|$

which can be used for plotting the curve shown in Fig. 4 . 


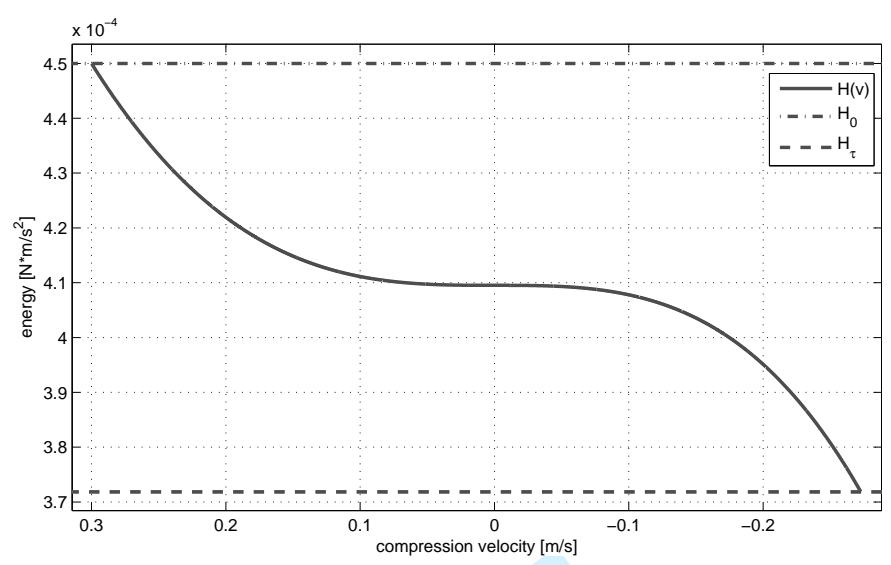

Fig. 4. Compression velocity-Hamiltonian characteristic. The two horizontal lines display $H_{0}=T_{0}$ and $H_{\tau}=T_{\tau}$, that is respectively the initial and the final Hamiltonian. The values of parameters are the same as in Fig. 1 for $v_{\text {in }}=1 \mathrm{~m} / \mathrm{s}$. The compression velocity axis has been inverted, thus allowing to read the graph from left to right.

\section{B. Addition of a constant external force}

When a constant external force $f_{e}$ (e.g. the force of gravity) is applied to the point mass, (2) has to be rewritten as:

$$
m a(t)+f_{e}=-f(x(t), v(t)) .
$$

Unfortunately, in this case no closed-form analytical results can be found as those described in II-A. In more detail, multiplying both sides of (21) by $v(t)$ and time-integrating them, an equation is found where an unsolvable integral is present, this way preventing to directly obtain explicit-form expressions for $x(v), \tilde{v}_{\text {out }}$ and $\tau$.

Rewriting the 2nd-order equation (21) as a system of 1 storder equations:

$$
\left\{\begin{array}{l}
\dot{x}=v \\
\dot{v}=-\frac{k}{m} x^{\alpha}-\frac{\lambda}{m} x^{\alpha} v-\frac{f_{e}}{m}
\end{array}\right.
$$

the equilibrium point of the system is found to be $\left(x_{\mathrm{eq}}, v_{\mathrm{eq}}\right)=$ $\left(-f_{e}^{1 / \alpha} / k, 0\right)$, which corresponds to the compression offset in stationary conditions.

As Fig. 5 shows, for positive values of $f_{e}$ and $v_{\text {in }}$, the velocity of the point mass during the compression phase is generally greater than in the case when $f_{e}=0$. In particular, at the beginning of contact interaction, since $f_{e}$ is higher than the current impact force $f$, the compression velocity exceeds $v_{\text {in }}$. On the other hand, compared to the case when $f_{e}=0$, during the decompression phase the absolute value of the point mass velocity $v$ decreases, resulting in lower output velocities. Moreover, the resulting maximum compression is always greater than that calculated by (4).

\section{NUMERICAL SIMULATIONS}

In this section, the continuous-time system described by (2) is discretized by means of several numerical methods, and the resulting numerical systems are studied.

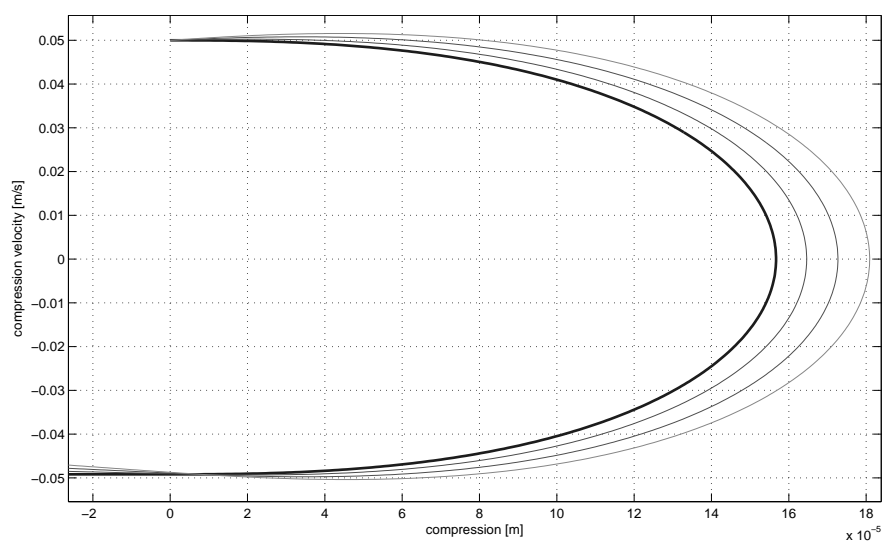

Fig. 5. Phase portraits of impacts for different external forces applied: $f_{e}=$ $m \cdot(1,2,3) \mathrm{N}$. The bold line represents the case where $f_{e}=0$. The values of parameters are the same as in Fig. 1 except for $k=10^{5} \mathrm{~N} / \mathrm{m}^{\alpha}$ and $v_{\text {in }}=0.05 \mathrm{~m} / \mathrm{s}$. Notice that, due to the lack of analytical results when an external force is present, the phase portraits have been obtained as the result of numerical simulations.

\section{A. Numerical methods}

Different numerical methods were considered, which are commonly used in various fields of applications spacing from computer graphics, to physical simulation of dynamical systems and digital signal processing. Thanks to their low order which generally results in low computational cost - the chosen methods are particularly suitable for real-time applications:

- The trapezoid rule is popular to translate analog filter structures to discrete-time filters, and it is the basis for wave digital filters;

- Verlet integration is popular in physics-based graphic engines;

- Heun is a 2nd-order method (complexity similar to the previous two) representative of the Runge-Kutta family;

- 4th-order Runge-Kutta is expected to be more accurate, and more expensive.

Following the standard notation in numerical analysis, the integration step is a constant named $h\left(=1 / F_{s}\right)$.

1) 1-step Adams-Moulton (AM1): is a A-stable 2nd-order implicit method [25], also known as bilinear transformation, or trapezoid rule.

Discretizing (2) results in the following equation in statespace form:

$$
\left[\begin{array}{l}
x_{n+1} \\
v_{n+1}
\end{array}\right]=\left[\begin{array}{cc}
1 & h \\
0 & 1
\end{array}\right]\left[\begin{array}{l}
x_{n} \\
v_{n}
\end{array}\right]+\left[\begin{array}{c}
\frac{h^{2}}{4 m} \\
\frac{h}{2 m}
\end{array}\right]\left[f_{n+1}+f_{n}\right]
$$

where the expression for the discrete-time force is obtained by replacing the continuous-time variables $x(t)$ and $v(t)$ in (1) with their discrete-time counterparts.

Since the AM1 method is implicit, 23) is also in implicit form, and this is reflected in the instantaneous relationship between $\left[\begin{array}{ll}x_{n+1} & v_{n+1}\end{array}\right]^{T}$ and $f_{n+1}$. Unfortunately, since $f_{n+1}$ also has an instantaneous dependence on $x_{n+1}$ and $v_{n+1}$ (given by (1)), the discrete-time counterpart of the system described by (2) contains a delay-free loop, which is not directly computable and - because of the nonlinear dependence $f(x, v)$ - needs some special handling in order to be solved. In particular, the K-method [26] together with Newton's 
method [25] are used, weighing on the efficiency of the simulation (see IV-C).

2) Verlet: is a 2nd-order explicit method commonly used in computer graphics [3], video games, and molecular dynamics simulation, where it is typically used for integrating Newton's equation of motion in order to describe the trajectory of moving particles. The one used here is a variant, called velocity Verlet, which provides better handling of the velocity variable and can be seen as a predictor-corrector method.

Discretizing (2), results in the following implementation scheme:

$$
\begin{aligned}
x_{n+1} & =x_{n}+h v_{n}+\frac{h^{2}}{2} \frac{f_{n}}{m}, \\
v_{n+\frac{1}{2}} & =v_{n}+\frac{h}{2} \frac{f_{n}}{m}, \quad \text { predictor, } \\
f_{n+1} & =f\left(x_{n+1}, v_{n+\frac{1}{2}}\right) \\
v_{n+1} & =v_{n+\frac{1}{2}}+\frac{h}{2} \frac{f_{n+1}}{m}, \quad \text { corrector. }
\end{aligned}
$$

It should be noted that this algorithm assumes that $f_{n+1}$ only depends on the predicted velocity $v_{n+\frac{1}{2}}$, which clearly gives rise to inaccuracies.

3) Heun: is a predictor-corrector explicit method [25], [3], with the forward Euler method as predictor and the trapezoid rule as corrector. It can also be seen as a 2 nd-order RungeKutta method (RK2).

Discretizing (2) results in the following implementation scheme:

$$
\begin{aligned}
\tilde{v}_{n+1} & =v_{n}+h \frac{f_{n}}{m}, \quad \text { predictor, } \\
x_{n+1} & =x_{n}+\frac{h}{2}\left(v_{n}+\tilde{v}_{n+1}\right), \\
f_{n+1} & =f\left(x_{n+1}, \tilde{v}_{n+1}\right), \\
v_{n+1} & =v_{n}+\frac{h}{2} \frac{f_{n}+f_{n+1}}{m}, \quad \text { corrector. }
\end{aligned}
$$

Again, it should be noted that both $x_{n+1}$ and $f_{n+1}$ only depend on the predicted velocity $\tilde{v}_{n+1}$, and this gives rise to inaccuracies.

4) 4th-order Runge-Kutta (RK4): is an explicit iterative method [25], [3] which is widely used to solve ODEs with improved accuracy.

Discretizing (2) results in the following implementation scheme:

$$
\begin{aligned}
& x_{n+1}=x_{n}+\frac{1}{6}\left(l_{1}+2 l_{2}+2 l_{3}+l_{4}\right) \\
& v_{n+1}=v_{n}+\frac{1}{6}\left(k_{1}+2 k_{2}+2 k_{3}+k_{4}\right)
\end{aligned}
$$

where:

$$
\begin{gathered}
l_{1}=h v_{n}, \quad l_{2}=h\left(v_{n}+\frac{k_{1}}{2}\right), \\
l_{3}=h\left(v_{n}+\frac{k_{2}}{2}\right), \quad l_{4}=h\left(v_{n}+k_{3}\right), \\
k_{1}=h \frac{f_{n}}{m}, \quad k_{2}=h \frac{f\left(x_{n}+\frac{l_{1}}{2}, v_{n}+\frac{k_{1}}{2}\right)}{m}, \\
k_{3}=h \frac{f\left(x_{n}+\frac{l_{2}}{2}, v_{n}+\frac{k_{2}}{2}\right)}{m}, \quad k_{4}=h \frac{f\left(x_{n}+l_{3}, v_{n}+k_{3}\right)}{m} .
\end{gathered}
$$

It should be noted that, for each sample, both the velocity and the non-linear force of (1) need to be evaluated four times, therefore strongly affecting the efficiency of the simulation (see IV-C).

\section{B. Experimental results}

In order to evaluate the chosen numerical methods, it is useful to compare the behavior of the corresponding simulations against the known analytical results (see $\amalg-\mathrm{A}$ ).

The main references used to quantitatively assess the reliability of a particular numerical method during contact are equations (3) and (20), which express respectively the compression $x$ and the Hamiltonian $H$ as functions of the compression velocity $v$. The errors on $x$ and $H$ are then measured as the maximum deviation $\sqrt{3}$ of their discrete-time versions from the respective analytical curves $x(v)$ and $H(v)$, in relation to the entire variation range of the quantities considered (i.e. $x_{\max }$ and $\Delta H_{\tau}$ ). In detail, taking into account 20 and defining $H^{\operatorname{sim}}\left(x_{n}, v_{n}\right)$ as the Hamiltonian computed using the values of compression and velocity resulting from a numerical simulation 4 and $\Delta H_{\tau}$ as in (18), the maximum deviation on $H$ is calculated by means of the following expression:

$$
\% \operatorname{dev}(H)=100 \cdot\left|\frac{\max _{n}\left\{H^{\operatorname{sim}}\left(x_{n}, v_{n}\right)-H\left(v_{n}\right)\right\}}{\Delta H_{\tau}}\right| .
$$

Considering (3) and defining $x_{\max }$ as in (4), the maximum deviation on $x$ is calculated as:

$$
\% \operatorname{dev}(x)=100 \cdot\left|\frac{\max _{n}\left\{x_{n}-x\left(v_{n}\right)\right\}}{x_{\max }}\right| .
$$

Finally, another indicator which allows to evaluate the accuracy and consistency of the simulations at release from contact is provided by the output velocity computed numerically as a zero of (6), which is used to calculate the relative error $\% \operatorname{err}\left(v_{\text {out }}\right)$ of its simulated counterpart $v_{\text {out }}^{\text {sim }}$.

Throughout the following example simulations some values of parameters are kept constant: $m=10^{-2} \mathrm{Kg}$, and $F_{s}=$ $44.1 \mathrm{kHz}$ (i.e. a standard audio sample rate).

1) Non-critical simulations: In order to verify the numerical implementations, the parameters of the model are set to a "safe" configuration, that is to far-from-extreme values. In this case, contact extends over many samples, thus ensuring that the simulations are influenced only to a negligible extent by the chosen sampling rate (see I-C) and should more likely behave as the original continuous-time system.

This is confirmed qualitatively by Fig. 6, where the plots of all such simulations substantially overlap and coincide with the analytical curves. Moreover, Table \ offers a quantitative evaluation of the simulations, showing the errors introduced by the different numerical methods considered.

\footnotetext{
${ }^{3}$ Such measures definitely have a different meaning compared to ordinary relative errors, since the latter would only account for local deviations from the analytical curves. On the other hand, the proposed measure of error is obtained normalizing the absolute error according to the range of variation of the quantities being evaluated, and picking the maximum deviation along such range.

${ }^{4}$ That is, substituting $x_{n}$ and $v_{n}$ in 13 . Not to be confused with the discrete Hamiltonian defined in [22] for finite-difference schemes.
} 
TABLE I

SUMMARY OF ERRORS IN NON-CRITICAL SIMULATIONS. THE VALUES OF PARAMETERS ARE $k=10^{3} \mathrm{~N} / \mathrm{M}^{\alpha}, \mu=0.5 \mathrm{~s} / \mathrm{M}, \alpha=1.5, v_{\mathrm{IN}}=0.5 \mathrm{M} / \mathrm{s}$.

\begin{tabular}{|l||c|c|c|c|}
\hline errors & AM1 & Verlet & Heun & RK4 \\
\hline$\% \operatorname{dev}(x)$ & 0.255 & 0.018 & 0.319 & 0.005 \\
$\% \operatorname{err}\left(v_{\text {out }}\right)$ & $+2 \cdot 10^{-5}$ & $+2 \cdot 10^{-6}$ & $-3 \cdot 10^{-5}$ & $+2 \cdot 10^{-6}$ \\
$\% \operatorname{dev}(H)$ & $3 \cdot 10^{-4}$ & 0.052 & $4 \cdot 10^{-4}$ & $1 \cdot 10^{-5}$ \\
\hline
\end{tabular}

2) Critical parameter regions: It has been found empirically that when $\tau^{\text {sim }} \leq 4$ samples, the errors on $x, H$ and $v_{\text {out }}$ heavily increase, resulting in an extremely poor reliability of all the simulations. The obvious reason for this behavior is that, since only very few samples of data are available, the numerical systems are totally unable to describe the original continuous-time counterpart. Hence, in the study hereafter, only values of parameters resulting in $\tau^{\text {sim }}>4$ samples are considered.

It has been observed that when $\mu \rightarrow 0_{+}$and/or as the contact time $\tau^{\text {sim }}$ decreases (i.e. for "hard" impacts), the behavior of most numerical implementations tends to become inconsistent with the continuous-time system. Hereafter, the numerical systems are studied for these two critical configurations, respectively named case 1 and case 2 .

Case 1: low dissipation $\left(\mu \rightarrow 0_{+}\right)$

In case of low dissipation, the Hamiltonian of both Verlet-

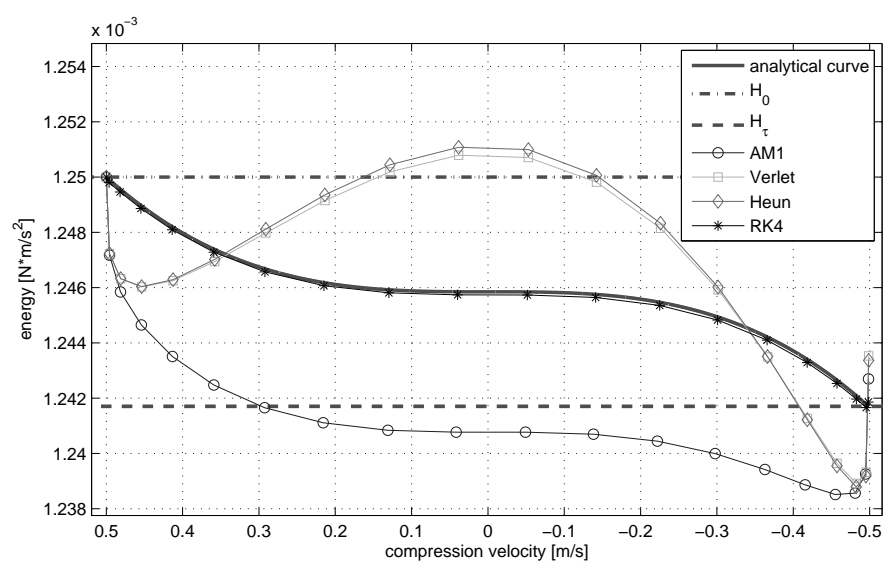

Fig. 7. Comparison of Hamiltonians for different implementations of a simulation example following case 1 . The values of parameters are $k=10^{7} \mathrm{~N} / \mathrm{m}^{\alpha}$, $\mu=0.01 \mathrm{~s} / \mathrm{m}, \alpha=1.3, v_{\text {in }}=0.5 \mathrm{~m} / \mathrm{s}$. The contact time equals 19 samples.

and Heun-discretized systems is prone to oscillations, while contact ends in an inconsistent final energy state: typically, $H_{\tau}^{\text {Verlet, Heun }}>H_{\tau}$, where $H_{\tau}$ is defined as in (17) and is calculated using values of $v_{\text {out }}$ computed numerically as zeros of (6). As for AM1-discretized systems, these generally tend to dissipate too much energy during contact (i.e. $H^{\mathrm{AM} 1}<H$ ), while slightly gaining spurious energy as the contact ends (i.e. $H_{\tau}^{\mathrm{AM} 1}>H_{\tau}$ ). On the other hand, RK4-discretized systems generally behave quite consistently both during and after the contact interaction (i.e. $H^{\mathrm{RK} 4} \approx H$ ).

Fig. 7 shows the Hamiltonian of a simulation example with low dissipation $(\mu=0.01)$, while Table 凹[a) shows the resulting errors.

\section{Case 2: hard impacts}

With the exception of RK4-discretized systems, in this case the simulations usually show more spread errors: $x^{\text {sim }}, H^{\text {sim }}$ and $v_{\text {out }}^{\text {sim }}$ tend to substantially deviate from the respective analytical results.

Fig. 8 shows a hard impact simulation example following the values of parameters adopted in Fig. 1 for $v_{i n}=1 \mathrm{~m} / \mathrm{s}$, while Table (b) shows the corresponding errors. The resulting contact time $\tau$ equals 6 samples.

It is worth noticing that the RK4 method has been proved to behave quite consistently across disparate configurations of parameters. Therefore, a highly oversampled RK-discretized system can be taken as a reference, able to provide extremely accurate simulations.

\section{IMPROVED NUMERICAL SIMULATIONS}

\section{A. Exploitation of analytical results}

In this section some solutions are proposed that allow to fix the inconsistencies pointed out in the accuracy and reliability of simulations which make use of the impact model under study, in view of their implementation as real-time applications. 


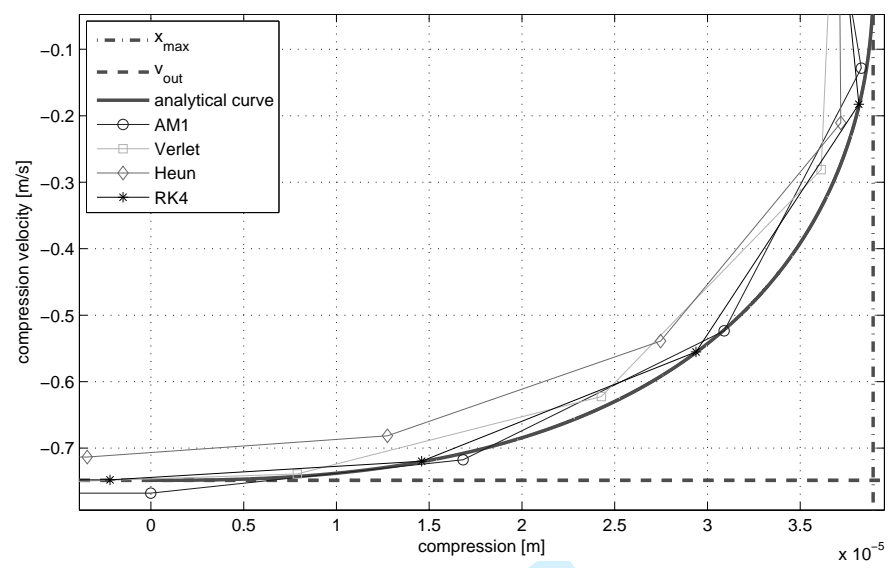

(a) Detail of phase portraits.

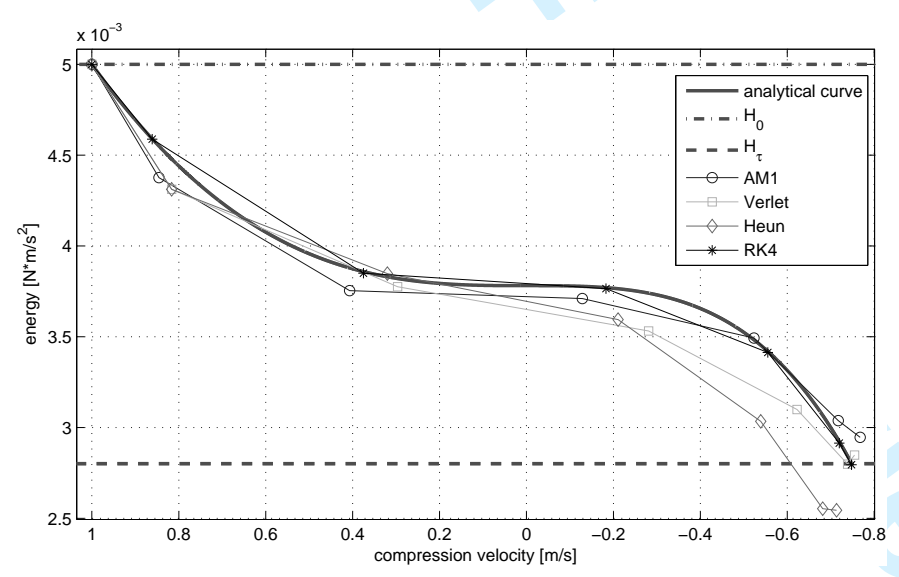

(b) Hamiltonians.

Fig. 8. Comparison of phase portraits and Hamiltonians for different implementations of a simulation example following case 2. The values of parameters are the same as in Fig. 1 with $v_{\text {in }}=1 \mathrm{~m} / \mathrm{s}$. The contact time equals 6 samples.

TABLE II

SUMMARY OF ERRORS IN EXAMPLE SIMULATIONS OF case 1 AND case 2. THE LAST COLUMN SHOWS THE ERROR YIELDED BY THE APPROXIMATE VALUE $\tilde{v}_{\text {OUT }}$ WITH RESPECT TO THE VALUE COMPUTED NUMERICALLY: IT IS WORTH NOTICING THAT IN BOTH CASES THE ERROR IS LOWER THAN THOSE YIELDED BY THE SIMULATIONS.

(a) Simulation example following case 1 . The values of parameters are the same as in Fig. 7

\begin{tabular}{|l||c|c|c|c|c|}
\hline errors & AM1 & Verlet & Heun & RK4 & $\tilde{v}_{\text {out }}$ \\
\hline$\% \operatorname{dev}(x)$ & 1.011 & 1.083 & 1.136 & 0.052 & - \\
$\% \operatorname{err}\left(v_{\text {out }}\right)$ & +0.039 & +0.073 & +0.067 & +0.006 & $-1 \cdot 10^{-5}$ \\
$\% \operatorname{dev}(H)$ & 61.302 & 59.542 & 63.042 & 1.427 & - \\
\hline
\end{tabular}

(b) Simulation example following case 2 . The values of parameters are the same as in Fig. 1 for $v_{\text {in }}=1 \mathrm{~m} / \mathrm{s}$.

\begin{tabular}{|l||c|c|c|c|c|}
\hline errors & AM1 & Verlet & Heun & RK4 & $\tilde{v}_{\text {out }}$ \\
\hline$\% \operatorname{dev}(x)$ & 4.381 & 4.418 & 19.506 & 0.412 & - \\
$\% \operatorname{err}\left(v_{\text {out }}\right)$ & +2.551 & +0.839 & -4.692 & -0.105 & -0.013 \\
$\% \operatorname{dev}(H)$ & 7.885 & 9.475 & 23.387 & 0.410 & - \\
\hline
\end{tabular}

1) Hybrid numerical-analytical computation: This solution consists in computing the compression velocity $v_{n}$ numerically - that is as a result of a numerical simulation, using for example one of the numerical methods described in II-A and employing it in (3) in order to calculate the corresponding value of compression $x\left(v_{n}\right)$ analytically. Different numerical methods may need different implementations of this solution.

As a result, the corrected numerical system strictly follows the analytical curves $x(v)$ and $H(v)$.

Since the computation is to be made at each sample for the whole duration of the contact interaction, this solution noticeably increases the computational load of the simulation.

2) Output velocity constraint: The solution above can be applied only while the contact interaction lasts, and therefore it cannot control the behavior of a numerical system upon the end of the impact. At that time, the energy content (i.e. the residual kinetic energy $T_{\tau}^{\text {sim }}=H_{\tau}^{\text {sim }}$ ) can be controlled by forcing the output velocity to the approximate value $\tilde{v}_{\text {out }}$.

Considering that the error introduced by (7) depends solely on the product $\mu v_{\text {in }}$ (see Fig. 3), it is advisable to apply the correction only when the product $\mu v_{\text {in }}$ corresponds to an acceptable error, or the risk is to even worsen the behavior of the numerical system. However, the use of this conditional correction always implies a trade-off: on the one hand it guarantees not to introduce errors greater than a chosen maximum, while on the other hand 1) within the excluded range of $\mu v_{\text {in }}$, the output velocity is not controlled and therefore depends only on the plain numerical method chosen, 2) within the included intervals of $\mu v_{\text {in }}$, the risk is that the correction introduces errors even greater than those provided by the noncorrected numerical system (this is true especially for noncritical parameter regions).

Once the output velocity has been forced to $\tilde{v}_{\text {out }}$, the corresponding compression should be set to 0 , this way adhering to the compression-force characteristics shown in Fig. 2 by closing their numerical counterpart at $(x=0, f=0)$, and ensuring that the final potential energy $V_{\tau}^{\text {sim }}$ is set to zero.

The computation of $\tilde{v}_{\text {out }}$ only needs to take place in correspondence to an impact event, and as soon as the impact velocity $v_{\text {in }}$ is known.

\section{B. Numerical simulations with corrections}

In order to test the described corrections, they were applied to the worst behaving simulation examples provided in 11 I-B2 Fig. 9 and 10 show a comparison of Heun-based simulations following respectively case 1 and case 2, with and without corrections.

1) Improved energy behavior: When the hybrid correction described in IV-A1 is applied, any simulation strictly adheres to the analytical curves $H(v)$ and $x(v)$ during contact, that is the respective errors, as defined in (27) and (28), are equal to zero 5

As for the energy state upon the end of the interaction, the error on $H_{\tau}$ depends either on the error introduced by $\tilde{v}_{\text {out }}$ (when the output velocity constraint described in IV-A2 is actually applied), or on the error introduced by the plain numerical simulation. As already stated in IV-A2 in the first case the maximum error on $v_{\text {out }}$ is predictable, and clearly the same goes for the error on $H_{\tau}$.

\footnotetext{
${ }^{5}$ Not taking into account the inherent errors related to the representation of numbers in the digital domain.
} 

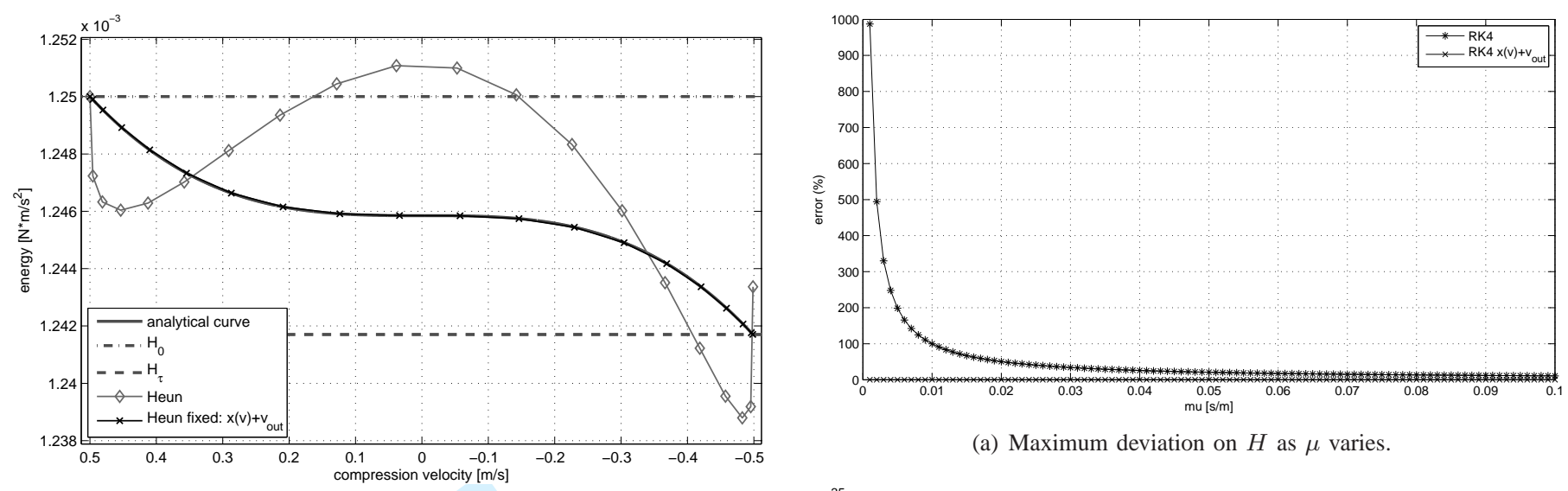

(a) Maximum deviation on $H$ as $\mu$ varies.

Fig. 9. Comparison of the Hamiltonians of a Heun-based simulation example following case 1, with and without corrections.

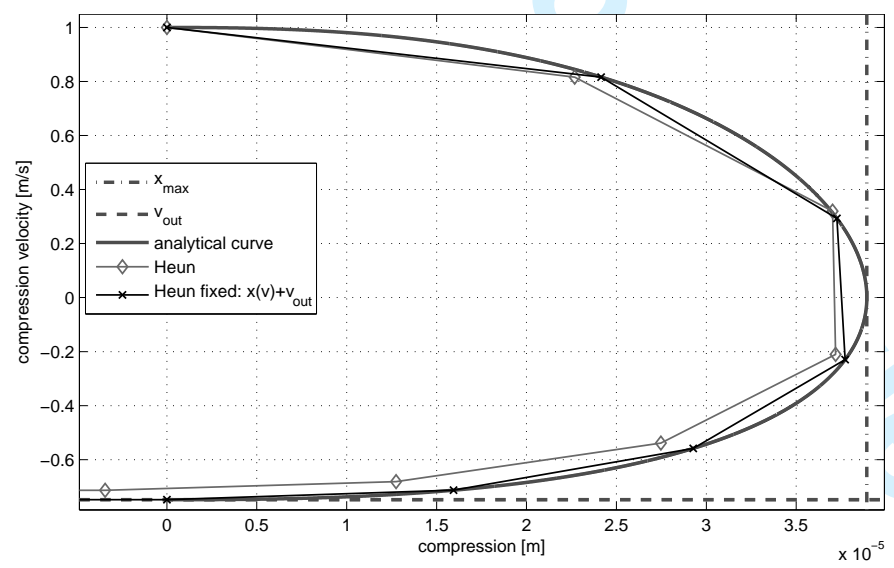

(a) Phase portraits.

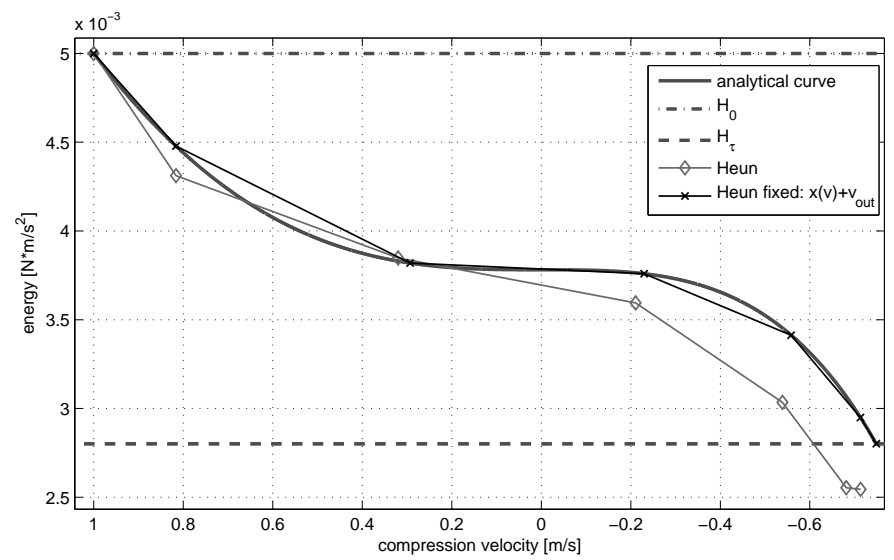

(b) Hamiltonians.

Fig. 10. Comparison of a Heun-based simulation example following case 2, with and without corrections.

Equation 27] allows to quantitatively assess the improvements on the energy consistency of the numerical simulations.

The trend of error on $H$ resulting from simulations with and without corrections is depicted in Figures 11(a) and 11(b), which show that even the best numerical method among those considered (i.e. RK4) can be improved, especially for critical values of parameters (see III-B2) such as $\mu v_{\text {in }} \rightarrow 0_{+}$.

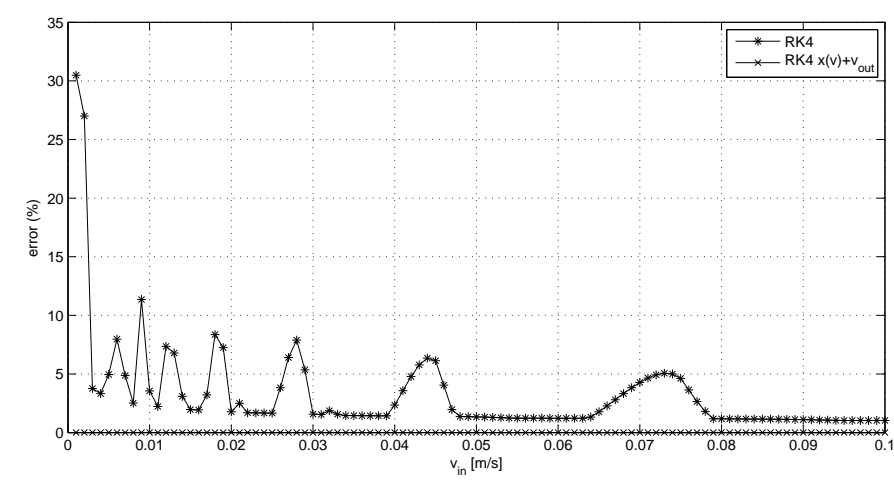

(b) Maximum deviation on $H$ as $v_{\text {in }}$ varies.

Fig. 11. Trend of error on the Hamiltonian for RK4-based simulations with and without corrections, for small values of $\mu$ and $v_{\text {in }}$. For the values of $\mu v_{\text {in }}$ considered, the output velocity constraint is always applied (see Fig. 3). The values of parameters, where kept constant, are $k=10^{9} \mathrm{~N} / \mathrm{m}^{\alpha}, \mu=0.5 \mathrm{~s} / \mathrm{m}$, $\alpha=1.5, v_{\text {in }}=0.5 \mathrm{~m} / \mathrm{s}$. At each corresponding point of the two parallel plots, the product $\mu v_{\text {in }}$ is the same. It can be noted that, when not using any corrections, the weight of $\mu$ on the error is clearly greater than that of $v_{\text {in }}$.

2) Sequence of impacts: In order to better appreciate the importance of the proposed corrections, a sequence of rebounds has been implemented applying a conservative force (e.g. gravity) during free motion only 6 Thanks to this setup, one can track the accumulation of energy anomalies at each contact interaction.

To this end, the residual energy $H_{\tau(i)}^{\text {sim }}$ of numerical simulations after the $i$-th impact was examined and compared to the residual energy $H_{\tau(i)}$ due to the exit velocity $v_{\text {out }}(i)$ of the $i$-th rebound, computed numerically as a zero of (6). The corresponding relative error provides a measure of the inaccuracies accumulated during the sequence of impacts. In addition, the deviation of $H^{\text {sim }}$ with respect to the analytical curve $H(v)$ was measured according to (27) along the whole sequence of impacts.

Table IIII shows the errors on the residual energy $H_{\tau(100)}^{\text {sim }}$ and the maximum deviations of $H^{\text {sim }}$ occurred during a sequence of 100 impacts, for simulation examples following case 1 and case 2 . Notice that, since in some simulations the energy can strongly oscillate during contact (see Fig. 7), the

\footnotetext{
${ }^{6}$ That is, the external force is temporarily suspended during the short contact at each rebound. As shown in contact, no closed-form analytical result is available, this way making the corrections provided in IV-A unsuitable.
} 


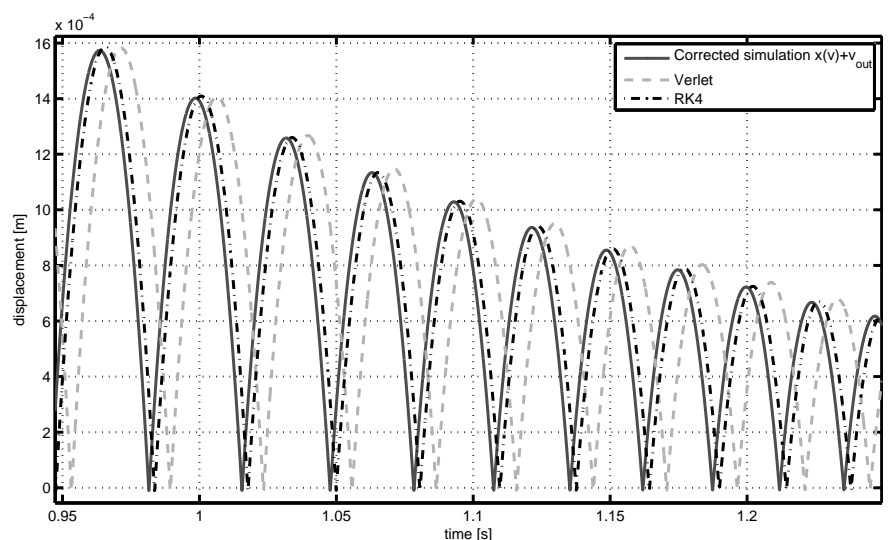

Fig. 12. Sequence of rebounds obtained from Verlet- and RK4-based simulations following case 2, compared with the trajectory of a corrected simulation.

maximum deviations from $H(v)$ do not necessarily reflect the accumulated errors. The last columns show the errors resulting from simulations corrected as suggested in IV-A where the error threshold for the output velocity constraint is set to $0.1 \%$, i.e. the correction is always applied (see Fig. 3).

\section{TABLE III}

SUMMARY OF ERRORS ON THE RESIDUAL ENERGY $H_{\tau(100)}^{\mathrm{SIM}}$ (ACCUMULATED ERROR), AND MAXIMUM DEVIATIONS OF $H^{\text {SIM }}$ CALCULATED ACCORDING TO 27 ALONG THE WHOLE SUCCESSION OF 100 REBOUNDS.

(a) Simulation examples following case 1 . The values of parameters are the same as in Fig. 7 except for input velocities decreasing at each rebound.

\begin{tabular}{|l||c|c|c|c|c|}
\hline errors on $H$ & AM1 & Verlet & Heun & RK4 & $\begin{array}{c}\text { corrected } \\
\text { sim. }\end{array}$ \\
\hline $\begin{array}{l}\text { accum. \%err } \\
\text { max \%dev }\end{array}$ & 12.022 & 10.059 & 147.036 & 0.907 & $<10^{-7}$ \\
0
\end{tabular}

(b) Simulation examples following case 2. The values of parameters are the same as in Fig. 1 starting with input velocity $v_{\text {in }}=1 \mathrm{~m} / \mathrm{s}$.

\begin{tabular}{|l||c|c|c|c|c|}
\hline errors on $H$ & AM1 & Verlet & Heun & RK4 & $\begin{array}{c}\text { corrected } \\
\text { sim. }\end{array}$ \\
\hline accum. \%err & 24.285 & 15.780 & 946.600 & 2.153 & 0.001 \\
max \%dev & 69.156 & 43.966 & 27.418 & 6.255 & 0 \\
\hline
\end{tabular}

In order to better understand the importance of such corrections, Fig. 12 provides a partial plot of the sequence of rebounds for case 2, where only the two better performing simulations are depicted.

From Table 11 and Fig. 12 it is evident that, even in case of errors apparently negligible for a single impact (see the errors relative to RK4 in Table (II), indeed energy inconsistencies accumulate and can become noticeable - if not disastrous after a certain number of contact interactions.

On the other hand, the errors resulting from the corrected simulations are actually negligible, and they are only due to inaccuracies in the forced exit velocity $\tilde{v}_{\text {out }}$. The effectiveness of such corrections is confirmed comparing the results of a $16 \times$ oversampled RK4 simulation used as a reference: in case $l$ the accumulated error on $H$ is $0.001 \%$ and the maximum deviation is $0.024 \%$, while in case 2 they are respectively $0.002 \%$ and $0.006 \%$.

\section{Computational cost}

In this section, the computational costs of both the numerical implementations seen in III-A and the corrections suggested in IV-A are taken into account. In particular, the cost has been measured as the number of operations (i.e., memory write/read accesses and arithmetical operations) needed to execute an algorithm.

Table IV(a) summarizes the cost of the algorithms implementing the numerical methods. Since the AM1-based implementation makes use of Newton's method, its cost is displayed on two sub-columns: the first one shows the constant cost per sample, while the second one (in italics) shows the cost of a single iteration of Newton's method. Notice that the number of iterations per sample is not predictable.

Table IV(b) shows the cost of the corrections considered. For comparison, the last column reports the cost of a single zero-finding iteration on (6). The number of iterations depends on $\mu$ and $v_{\text {in }}$, and is usually in the order of some tens 7 It is clear that, despite being more precise than the approximate value $\tilde{v}_{\text {out }}$, the value computed numerically as a zero of (6) implies several times the number of operations required by $\tilde{v}_{\text {out }}$.

Since the computational load of simple write/read operations is generally low (if not negligible), two totals for each column are reported: one excluding write/read operations and, in brackets, one accounting for them.

\section{TABLE IV}

NUMBER OF OPERATIONS NEEDED BY THE NUMERICAL METHODS SHOWN IN III-A AND THE CORRECTIONS DESCRIBED INIV-A THE TOTALS IN BRACKETS ACCOUNT FOR WRITE/READ OPERATIONS.

(a) Since the AM1-based implementation makes use of Newton's method, its cost is displayed on two sub-columns: the first one shows the cost per sample, while the second one (in italics) shows the cost of a single Newton's method iteration.

\begin{tabular}{|l||cc|c|c|c|}
\hline \multicolumn{1}{|c||}{} & \multicolumn{2}{c|}{ AM1 } & Verlet & Heun & RK4 \\
\hline write & 6 & 10 & 5 & 5 & 20 \\
read & 16 & 33 & 20 & 21 & 72 \\
\hline$+l-$ & 3 & 8 & 7 & 8 & 22 \\
$\times$ & 8 & 11 & 5 & 5 & 18 \\
$\div$ & 0 & 1 & 3 & 3 & 8 \\
bit-shift & 0 & 0 & 2 & 3 & 6 \\
exp & 0 & 1 & 1 & 1 & 4 \\
log & 0 & 0 & 0 & 0 & 0 \\
compare & 0 & 4 & 1 & 1 & 4 \\
\hline TOTAL & $11(33)$ & $25(68)$ & $19(44)$ & $21(47)$ & $62(154)$ \\
\hline
\end{tabular}

(b) The last column (in italics) shows the cost of a single zero-finding iteration on 6 .

\begin{tabular}{|l||c|c|c|}
\hline & $\begin{array}{c}\text { hybrid } \\
\text { correct. }\end{array}$ & $\begin{array}{c}\tilde{v}_{\text {out }} \\
\text { constraint }\end{array}$ & $\begin{array}{c}v_{\text {out }} \\
\text { as zero of [6 }\end{array}$ \\
\hline write & 1 & 2 & 1 \\
read & 7 & 4 & 3 \\
\hline$+/-$ & 6 & 5 & 4 \\
$\times$ & 7 & 6 & 3 \\
$\div$ & 3 & 4 & 1 \\
bit-shift & 0 & 1 & 0 \\
exp & 0 & 1 & 0 \\
log & 1 & 0 & 1 \\
compare & 1 & 1 & 0 \\
\hline TOTAL & $18(26)$ & $18(24)$ & $9(12)$ \\
\hline
\end{tabular}

${ }^{7}$ The number of iterations was empirically found as being usually between 15 and 40 . Moreover, as $\mu$ decreases, the number of iterations increases. 
Recalling that the hybrid correction only affects the computational cost during contact, whereas the output velocity constraint is applied at mos 8 once per impact event, from Table IV one can infer that Verlet- or Heun-based simulations with corrections are roughly three times as efficient as plain RK4-based simulations during free motion, and almost twice as efficient during contact. On the other hand, while the exact computational load of an AM1-based simulation is not predictable, it can be noted that it already matches the cost of a RK4-based one after two iterations of Newton's method.

\section{Evaluation of methods}

The considered implementations can be finally evaluated in the light of the results regarding their accuracy (see [II-B and IV-B2) and computational load (see IV-C).

It can be stated that, among the non-corrected implementations, the best all-round performance is achieved by RK4discretized systems, although they are quite computationally expensive. On the other hand, a corrected Verlet-discretized system is generally at least as good as a non-corrected RK4 implementation, at a fraction of its computational load.

As for AM1-based implementations, it was shown that they behave quite poorly in the critical regions identified as case 1 and case 2. These poor results in terms of accuracy, together with a generally high (and non-predictable) computational load, set the AM1 method as a hardly recommendable choice. Apparently such conclusion can be quite surprising, especially if one considers that AM1 is the only implicit and $A$-stable method among those considered. However, the presence of nonlinearities, together with the inaccuracies introduced by the K-method and Newton's method, justify the behavior of AM1based implementations.

\section{Conclusions}

A non-linear physical model of impact with sound synthesis applications has been reviewed, and its properties have been studied using both analytical tools and numerical simulations.

Several numerical realizations have been compared, and their shortcomings with regard to the corresponding analytical results have been pointed out. Special emphasis has been placed on energy consistency.

It has been shown that by exploiting the analytical results provided, the inconsistencies of the numerical realizations can be amended, thus restoring the correct energy state of the simulated systems, during and after contact.

Future research will consider finding a closed-form approximation of the release velocity for the system of (21), where a constant external force is applied, in this way allowing to implement suitable corrections. Even without such a closedform solution, zero-finding numerical procedures could be profitably used to fix individual impacts. More interestingly for applications in acoustics, solutions will be investigated for extending the corrections to the case where vibrational losses are present, thus being applicable to impacts with resonating objects.

${ }^{8}$ As shown in IV-A2 the output velocity constraint is applied conditionally.

\section{APPENDIX}

\section{A. Polynomial expansion of the output velocity}

As mentioned in [I-A2, Hunt and Crossley [12] suggested that, in the limit $\mu v_{\text {in }} \rightarrow 0_{+}$, the restitution coefficient can be approximated by the linear function $\widetilde{E}\left(\mu v_{\text {in }}\right)=\left(1-2 / 3 \mu v_{\text {in }}\right)$. Then, recalling (5), the corresponding output velocity is:

$$
\tilde{v}_{\text {out }}\left(\mu, v_{\text {in }}\right)=\left(-v_{\text {in }}+\frac{2}{3} \mu v_{\text {in }}^{2}\right) \text {. }
$$

This result can be easily verified through a Taylor expansion of the two sides in (6), using the approximation $\log |1+\epsilon| \approx$ $\epsilon-\frac{\epsilon^{2}}{2}+\frac{\epsilon^{3}}{3}$, for $\epsilon \rightarrow 0_{+}$.

The same approach can be used in order to find a polynomial expansion of order $n$ :

$$
\tilde{v}_{\text {out }}=\sum_{j=1}^{n} a_{j} \cdot v_{\text {in }}^{j}
$$

in the limit $\mu v_{\text {in }} \rightarrow 0_{+}$. Equation (30) is then substituted into the left-hand side of the Taylor expansion of (6). For the case $n=4$ one obtains:

$$
\begin{aligned}
& {\left[\frac{\mu^{2}}{2} v_{\text {out }}^{2}-\frac{\mu^{3}}{3} v_{\text {out }}^{3}+\frac{\mu^{4}}{4} v_{\text {out }}^{4}-\frac{\mu^{5}}{5} v_{\text {out }}^{5}\right]_{v_{\text {out }}=\sum_{1}^{4} a_{j} v_{\text {in }}^{j}}} \\
& =\frac{1}{2} \mu^{2} v_{\text {in }}^{2}-\frac{1}{3} \mu^{3} v_{\text {in }}^{3}+\frac{1}{4} \mu^{4} v_{\text {in }}^{4}-\frac{1}{5} \mu^{5} v_{\text {in }}^{5} .
\end{aligned}
$$

The coefficients $a_{j}$ are then determined by equating the two sides of 31 term by term, leading to the system:

$$
\left\{\begin{array}{l}
\frac{1}{2} \mu^{2} a_{1}^{2}=\frac{1}{2} \mu^{2}, \\
\frac{1}{3} \mu^{3} a_{1}^{3}-\mu^{2} a_{1} a_{2}=\frac{1}{3} \mu^{2} \\
\frac{1}{4} \mu^{4} a_{1}^{4}-\mu^{3} a_{1}^{2} a_{2}+\mu^{2} a_{1} a_{3}+\frac{1}{2} \mu^{2} a_{2}^{2}=\frac{1}{4} \mu^{2}, \\
\frac{1}{5} \mu^{5} a_{1}^{5}-\mu^{4} a_{1}^{3} a_{2}+\mu^{3} a_{1} a_{2}^{2}+ \\
\quad+\mu^{3} a_{1}^{2} a_{3}-\mu^{2} a_{1} a_{4}-\mu^{2} a_{2} a_{3}=\frac{1}{5} \mu^{2}
\end{array}\right.
$$

which yields:

$$
a_{1}=-1, \quad a_{2}=\frac{2}{3} \mu, \quad a_{3}=-\frac{4}{9} \mu^{2}, \quad a_{4}=\frac{44}{135} \mu^{3} .
$$

It follows that, in (30), $v_{\text {out }}$ is a function of $\left(\mu, v_{\text {in }}\right)$ only. Equations (30) and (33) result in a slightly better approximation than (29), but still provide a local approximation.

\section{B. A non-local approximation for the output velocity}

From (6) $v_{\text {out }}$ can be written as:

$$
\begin{aligned}
v_{\text {out }} & =\frac{1}{\mu}\left[\left(1+\mu v_{\text {in }}\right) e^{-\mu \Delta v}-1\right]= \\
& =v_{\lim }\left[1-\left(1+\mu v_{\text {in }}\right) e^{-\mu \Delta v}\right]
\end{aligned}
$$

where $\Delta v=v_{\text {in }}-v_{\text {out }}$. Equation (34) emphasizes that the convergence $v_{\text {out }} \rightarrow v_{\text {lim }}$ for $v_{\text {in }} \rightarrow+\infty$ is governed by the fast-decreasing term $e^{-\mu \Delta v}$. 
For small $v_{\text {in }}$ 's, the zeroth order approximation of the exponential term is $e^{-2 \mu v_{\text {in }}}$, however it is easy to verify that the equation:

$$
\tilde{v}_{\text {out }}=v_{\text {lim }}\left[1-\left(1+\mu v_{\text {in }}\right) e^{-2 \mu v_{\text {in }}}\right]
$$

does not provide an accurate approximation of $v_{\text {out }}$ for small $v_{\text {in }}$ 's. More precisely, its Taylor expansion around $v_{\text {in }}=0$ only matches the first Taylor coefficient of the expansion (30):

$$
\begin{aligned}
\tilde{v}_{\text {out }} \underset{v_{\text {in }} \rightarrow 0_{+}}{\underset{\sim}{\approx}} \sum_{j=1}^{n} \tilde{a}_{j} \cdot v_{\text {in }}^{j}, \\
\tilde{a}_{1}=-1 \quad \text { and } \quad \tilde{a}_{j} \neq a_{j} \quad(j>1) .
\end{aligned}
$$

This qualitative discussion justifies to some extent the nonlocal approximation for $v_{\text {out }}$ given in (7). Indeed (7) combines the two views $-\mu v_{\text {in }} \rightarrow 0_{+}$and $\mu v_{\text {in }} \rightarrow+\infty-$ on $v_{\text {out }}$ : the exponential term ensures the convergence $v_{\text {out }} \rightarrow v_{\text {lim }}$ for high values of $\mu v_{\text {in }}$, while the polynomial coefficients $b_{j}$ are determined by imposing that the Taylor expansion of (7) around $v_{\text {in }}=0$ matches that of (30). Recalling that:

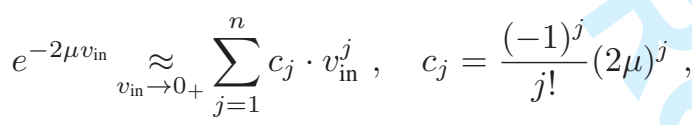

then one can verify that the $l$-th order coefficient $a_{l}$ of the Taylor expansion of (7) is given as $a_{l}=\frac{1}{\mu} \sum_{j=1}^{l} b_{j} \cdot c_{(l-j)}$. Then the coefficients $b_{j}$ can be determined recursively as:

$$
b_{0}=1, \quad b_{j}=\mu a_{j}-\sum_{l=0}^{j-1} b_{l} \cdot c_{(j-l)} \quad(1 \leq j \leq n) .
$$

Applying this recursive equation for the case $n=4$ yields $(8)$.

\section{REFERENCES}

[1] D. W. Marhefka and D. E. Orin, "A compliant contact model with nonlinear damping for simulation of robotic systems," IEEE Trans. on Systems, Man, and Cybernetics - Part A: Systems and Humans, vol. 29, no. 6, pp. 566-572, November 1999.

[2] M. Mahvash, V. Hayward, and J. Lloyd, "Haptic rendering of tool contact," in Proc. Eurohaptics, 2002, pp. 110-115.

[3] M. Müller, J. Stam, D. James, and N. Thürey, "Real time physics: class notes," in SIGGRAPH '08: ACM SIGGRAPH 2008 classes. New York, NY, USA: ACM, 2008, pp. 1-90.

[4] A. Chaigne and J. Kergomard, Acoustique des Instruments de Musique. Belin, 2008

[5] F. Avanzini and D. Rocchesso, "Modeling collision sounds: non-linear contact force," in Proc. Int. Conf. on Digital Audio Effects (DAFx-01), Limerick, December 2001, pp. 61-66.

[6] M. Rath and D. Rocchesso, "Continuous sonic feedback from a rolling ball," IEEE MultiMedia, vol. 12, no. 2, pp. 60-69, 2005.

[7] D. Rocchesso and F. Fontana, Eds., The Sounding Object. Mondo Estremo, 2003. [Online]. Available: http://www.soundobject.org/

[8] P. Flores, J. P. Claro, and H. M. Lankarani, Kinematics and Dynamics of Multibody Systems with Imperfect Joints: Models and Case Studies. Springer, 2008.

[9] G. Kuwabara and K. Kono, "Restitution coefficient in a collision between two spheres," Jap. J. of Appl. Phys., vol. 26, no. 8, pp. 12301233, 1987.

[10] A. Stulov, "Dynamic behavior and mechanical features of wool felt," Acta Mechanica, vol. 169, no. 1, pp. 13-21, 2004.

[11] L. Vu-Quoc and X. Zhang, "An elastoplastic contact force-displacement model in the normal direction: displacement-driven version," Proc.: Mathematical, Physical and Engineering Sciences, pp. 4013-4044, 1999.

[12] K. H. Hunt and F. R. E. Crossley, "Coefficient of restitution interpreted as damping in vibroimpact," ASME J. Applied Mech., pp. 440-445, June 1975.
[13] N. Diolaiti, C. Melchiorri, and S. Stramigioli, "Contact impedance estimation for robotic systems," IEEE Trans. on Robotics, vol. 21, no. 5, pp. 925-935, 2005.

[14] H. M. Lankarani and P. E. Nikravesh, "A contact force model with hysteresis damping for impact analysis of multibody systems," $J$. of Mechanical Design, vol. 112, no. 3, pp. 369-376, 1990.

[15] P. Flores, J. Ambrósio, J. Claro, and H. Lankarani, "Influence of the contact-impact force model on the dynamic response of multi-body systems," Proc. of the Institution of Mechanical Engineers-K, vol. 220, no. 1, pp. 21-34, 2006.

[16] L. Pust and F. Peterka, "Impact oscillator with Hertz's model of contact," Meccanica, vol. 38, no. 1, pp. 99-116, 2003.

[17] S. Papetti, F. Fontana, M. Civolani, A. Berrezag, and V. Hayward, "Audio-tactile display of ground properties using interactive shoes," in Proc. 5th Int. Workshop on Haptic and Audio Interaction Design (HAID '10), Copenhagen, Denmark, 2010, accepted.

[18] S. Bilbao, "Robust physical modeling sound synthesis for nonlinear systems," Signal Processing Magazine, IEEE, vol. 24, no. 2, pp. $32-$ 41, March 2007.

[19] I. Millington, Game physics engine development. Morgan Kaufmann, 2007.

[20] J. E. Colgate and J. M. Brown, "Factors Affecting the Z-Width of a Haptic Display," in Proc. IEEE Int. Conf. on Robotics \& Automation, San Diego, May 1994, pp. 3205-3210.

[21] K. Kuchenbecker, J. Fiene, and G. Niemeyer, "Improving contact realism through event-based haptic feedback," IEEE Trans. on Visualization and Computer Graphics, vol. 12, no. 2, pp. 219-230, March-April 2006.

[22] S. Bilbao, Numerical Sound Synthesis. New York: Wiley, 2009.

[23] F. Avanzini and D. Rocchesso, "Physical modeling of impacts: theory and experiments on contact time and spectral centroid," in Proc. Int. Conf. on Sound and Music Computing (SMC'04), 2004, pp. 287-293.

[24] B. L. Giordano, D. Rocchesso, and S. McAdams, "Integration of acoustical information in the perception of impacted sound sources: The role of information accuracy and exploitability," Journal of Experimental Psychology: Human Perception and Performance, vol. 36, no. 2, pp. 462-476, 2010.

[25] A. Quarteroni, R. Sacco, and F. Saleri, Numerical Mathematics, 2nd ed. Springer, 2007.

[26] G. Borin, G. De Poli, and D. Rocchesso, "Elimination of Delay-Free Loops in Discrete-Time Models of Nonlinear Acoustic Systems," IEEE Trans. on Speech and Audio Processing, vol. 8, no. 5, pp. 597-605, September 2000. 
To ensure uniformity of treatment among all contributors, other forms may not be substituted for this form, nor may any wording of the form be changed. This form is intended for original material submitted to the IEEE and must accompany any such material in order to be published by the IEEE. Please read the form carefully and keep a copy for your files.

\section{TITLE OF PAPER/ARTICLE/REPORT, INCLUDING ALL CONTENT IN ANY FORM, FORMAT, OR MEDIA (hereinafter, "the Work"):}

\section{COMPLETE LIST OF AUTHORS:}

IEEE PUBLICATTON TTYLE (Journal, Magazine, Conference, Book):

\section{Copyright Transfer}

1. The undersigned hereby assigns to The Institute of Electrical and Electronics Engineers, Incorporated (the "IEEE") all rights under copyright that may exist in and to: (a) the above Work, including any revised or expanded derivative works submitted to the IEEE by the undersigned based on the Work; and (b) any associated written or multimedia components or other enhancements accompanying the Work.

\section{Consent and Release}

2. In the event the undersigned makes a presentation based upon the Work at a conference bosted or sponsored in whole or in part by the IEEE, the undersigned, in consideration for his/her participation in the conference, hereby grants the IREE the unlimited, worldwide, irrevocable permission to use, distribute, publish, license, exhibit, record, digitize, broadcast, reproduce and archive, in any format or medium, whether now known or hereafter developed: (a) his/her presentation and comments at the conference; (b) any written materials or multimedia files used in connection with his/her presentation; and (c) any recorded interviews of him/her (collectively, the "Presentation"). The permission granted includes the transcription and reproduction of the Presentation for inclusion in products sold or distributed by IEEE and live or recorded broadcast of the Presentation during or after the conference.

3. In connection with the permission granted in Section 2, the undersigned hereby grants IEEE the unlimited, worldwide, irrevocable right to use his/her name, picture, likeness, voice and biographical information as part of the advertisement, distribution and sale of products incorporating the Work or Presentation, and releases IEEE from any claim based on right of privacy or publicity.

4. The undersigned hereby warrants that the Work and Presentation (collectively, the "Materials") are original and that he/she is the author of the Materials, To the extent the Materials incorporate text passages, figures, data or other material from the works of others, the undersigned has obtained any necessary permissions. Where necessary, the undersigned has obtained ail third party permissions and consents to grant the license above and has provided copies of such permissions and consents to IEEE.

Please check this box if you do not wish to have video/audio recordings made of your conference presentation.

See reverse side for Retained Rights/Terms and Conditions, and Author Responsibilities.

\section{General Terms}

- The undersigned represents that he/she has the power and authority to make and execute this assignment.

- The undersigned agrees to indemnify and hold harmless the EEE from any damage or expense that may arise in the event of a breach of any of the warranties set forth above.

- In the event the above work is not accepted and published by the IEEE or is withdrawn by the author(s) before acceptance by the IEEE, the foregoing copyright transfer shall become null and void and all materials embodying the Work submitted to the IEEE will be destroyed.

- For jointly authored Works, all joint authors should sign, or one of the authors should sign as authorized agent for the others.

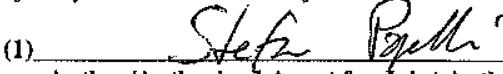
Author/Authorized Agent for Joint Authors

Nov. Noth 2oog

\section{U.S. Government Employee Certification (where applicable)}

This will certify that all authors of the Work are U.S. government employees and prepared the Work on a subject within the scope of their official dutics. As such, the Work is not subject to U.S. copyright protection.

(2)

Authorized Signature

Date

(Authors who are U.S. government employees should also sign signature line (1) above to enable the IEEE to claim and protect its copyright in international jurisdictions.)

\section{Crown Copyright Certification (where applicable)}

This will certify that all authors of the Work are employees of the British or British Commonwealth Government and prepared the Work in connection with their official duties. As such, the Work is subject to Crown Copyright and is not assigned to the IEEE as set forth in the first sentence of the Copyright Transfer Section above. The undersigned acknowledges, however, that the IEEE has the right to publish, distribute and reprint the Work in all forms and media.

(3)

Authorized Signature

Date

(Authors who are British or British Commonwealth Government employees should also sign line (1) above to indicate their acceptance of all terms other than the copyright transfer.) 


\section{IEEE COPYRIGHT FORM (continued)}

\section{RETAINED RIGHTS/TERMS AND CONDITIONS}

1. Authors/employers retain all proprietary rights in any process, procedure, or article of manufacture described in the Work.

2. Authors/employers may reproduce or authorize others to reproduce the Work, material extracted verbatim from the Work, or derivative works for the author's personal use or for company use, provided that the source and the IEEE copyright notice are indicated, the copies are not used in any way that implies IEEE endorsement of a product or service of any employer, and the copies themselves are not offered for sale.

3. Authors/employers may make limited distribution of all or portions of the Work prior to publication if they inform the IEEE in advance of the nature and extent of such limited distribution.

4. In the case of a Work performed under a U.S. Government contract or grant, the IEEE recognizes that the U.S. Government has royalty-free permission to reproduce all or portions of the Work, and to authorize others to do so, for official U.S. Government purposes only, if the contract/grant so requires.

5. For all uses not covered by items 2,3, and 4, authors/employers must request permission from the IEEE Intellectual Property Rights office to reproduce or authorize the reproduction of the Work or material extracted verbatim from the Work, including figures and tables.

6. Although authors are permitted to re-use all or portions of the Work in other works, this does not include granting third-party requests for reprinting, republishing, or other types of re-use. The IEEE Intellectual Property Rights office must handle all such third-party requests.

\section{INFORMATION FOR AUTHORS}

\section{Author Responsibilities}

The IEEE distributes its technical publications throughout the world and wants to ensure that the material submitted to its publications is properly available to the readership of those publications. Authors must ensure that their Work meets the requirements as stated in section 8.2.1 of the IEEE PSPB Operations Manual, including provisions covering originality, authorship, author responsibilities and author misconduct. More information on IEEE's publishing policies may be found at http://www.ieee,org/web/publications/pubtoolsandpolicyinfo/index.html. Authors are advised especially of IEEE PSPB Operations Manual section 8.2.1.B12: "It is the responsibility of the authors, not the IEEE, to determine whether disclosure of their material requires the prior consent of other parties and, if so, to obtain it." Authors are also advised of IEEE PSPB Operations Manual section 8.1.1B: "Statements and opinions given in work published by the IEEE are the expression of the authors."

\section{Author/Employer Rights}

If you are employed and prepared the Work on a subject within the scope of your employment, the copyright in the Work belongs to your employer as a work-for-hire. In that case, the IEEE assumes that when you sign this Form, you are authorized to do so by your employer and that your employer has consented to the transfer of copyright, to the representation and warranty of publication rights, and to all other terms and conditions of this Form. If such authorization and consent has not been given to you, an authorized representative of your employer should sign this Form as the Author.

\section{IEEE Copyright Ownership}

It is the formal policy of the IEEE to own the copyrights to all copyrightable material in its technical publications and to the individual contributions contained therein, in order to protect the interests of the IEEE, its authors and their employers, and, at the same time, to facilitate the appropriate reuse of this material by others. The IEEE distributes its technical publications throughout the world and does so by various means such as hard copy, microfiche, microfilm, and electronic media. It also abstracts and may translate its publications, and articles contained therein, for inclusion in various compendiums, collective works, databases and similar publications.

\section{Reprint/Republication Policy}

The IEEE requires that the consent of the first-named author and employer be sought as a condition to granting reprint or republication rights to others or for permitting use of a Work for promotion or marketing purposes.

THIS FORM MUST ACCOMPANY THE SUBMISSION OF THE AUTHOR'S MANUSCRIPT.

Questions about the submission of the form or manuscript must be sent to the publication's editor. Please direct all questions about IEEE copyright policy to:

IEEE Intellectual Property Rights Office, copyrights@ieee.org, +1-732-562-3966 (telephone) 